\title{
A Systematic Review of Socioeconomic Indicators and Dental Caries in Adults
}

Simone M. Costa ${ }^{1}$, Carolina C. Martins ${ }^{1}$, Maria de Lourdes C. Bonfim ${ }^{1}$, Lívia G. Zina ${ }^{2}$, Saul M. Paiva ${ }^{1}$, Isabela A. Pordeus ${ }^{1}$ and Mauro H. N. G. Abreu ${ }^{1, *}$

1 School of Dentistry, Universidade Federal de Minas Gerais, Belo Horizonte 31270-901, Brazil; E-Mails: smelocosta@gmail.com (S.M.C.); carolcm10@hotmail.com (C.C.M.); malu_cb2000@yahoo.com.br(M.L.C.B.); smpaiva@uol.com.br (S.M.P.); isabelapordeus@ufmg.br (I.A.P.)

2 Minas Gerais State Public Health School, Avenida Augusto de Lima, 2061-Barro Preto, Belo Horizonte MG 30190-002, Brazil; E-Mail: liviazina@yahoo.com.br

* Author to whom correspondence should be addressed; E-Mail: maurohenriqueabreu@ig.com.br; Tel./Fax: +55-31-3409-2442.

Received: 28 May 2012; in revised form: 10 September 2012 / Accepted: 2 October 2012 / Published: 10 October 2012

\begin{abstract}
Increasing evidence suggests that socioeconomic factors may be associated with an increased risk of dental caries. To provide better evidence of the association between dental caries in adults and socioeconomic indicators, we evaluated the relation between these two conditions in a thorough review of the literature. Seven databases were systematically searched: Pubmed, Cochrane, Web of Science, Bireme, Controlled Trials, Clinical Trials and the National Institute for Health and Clinical Excellence. No restrictions were placed on the language or year of publication. The search yielded 41 studies for systematic review. Two independent reviewers screened the studies for inclusion, extracted data and evaluated quality using the Newcastle-Ottawa scale. The following socioeconomic indicators were found: educational level, income, occupation, socioeconomic status and the community index. These indicators were significantly associated with a greater occurrence of dental caries: the subject's education, subject's income, subject's occupation and the Gini coefficient. A high degree of heterogeneity was found among the methods. Quality varied across studies. The criteria employed for socioeconomic indicators and dental caries should be standardized in future studies. The scientific evidence reveals that educational level, income, occupation and the Gini coefficient are associated with dental caries.
\end{abstract}


Keywords: epidemiology; caries; adults

\section{Introduction}

There has been a reduction in the prevalence of dental caries in both developed and developing countries [1]. However, the prevalence remains high among populations of low socioeconomic status. Therefore, socioeconomic indicators are associated with risk factors for dental caries [2,3]. Socially disadvantaged individuals also experience disadvantages with regard to health in general. The greater frequencies of disease in small population groups are known as polarization [4,5]. The association between the relative position each social group occupies and differences in the risk for various health conditions and in access to healthcare services makes social stratification a determinant of these conditions.

Social epidemiology has made great advances over the past three decades at a time when health inequalities have widened across countries. This situation challenges researchers to understand the social disparities in health to improve population health [6], despite the need to generate improved theoretical frameworks and the necessary data to test and refine them [7]. Recently, it was reinforced that social class or socioeconomic position, are not only a striking predictor of disease occurrence, but the associations reflects causal connections too [8].

The causal approach in dental caries was previously presented. Low socioeconomic status, low monthly household income and low educational level are associated with less access to dental services and oral hygiene products, poorer knowledge regarding oral health and oral hygiene and, consequently, a greater frequency and severity of dental caries [9].

Although a number of epidemiological studies have evaluated the associations between dental caries and socioeconomic indicators, no systematic reviews in the literature offer scientific evidence of such associations. The aim of the present study was to perform a systematic review to evaluate the associations between socioeconomic indicators and dental caries in adults by narrative synthesis. The hypothesis was that adults with worse socioeconomic indicators are more affected by dental caries.

\section{Methods}

All epidemiological studies (cross-sectional, case-control, cohort and clinical trials) involving adult populations aged 19 to 60 years that reported etiological factors and/or the prevalence of dental caries or risk factors for dental caries were considered eligible for the present review. Study selection was conducted in two phases: (1) abstracts and titles were selected and (2) full texts of the selected titles were obtained and read to determine the final sample set.

The epidemiological question investigated in this study was as follows: are adults with worse socioeconomic indicators more affected by dental caries than adults with better socioeconomic indicators? The socioeconomic indicators included any reference to schooling, income, type of occupation or employment, socioeconomic status, any population index, access/non-access to private dental practice and satisfaction with one's income. 


\subsection{Search Strategy}

Seven databases were systematically searched: MEDLINE using PubMed (www.pubmed.gov), The Cochrane Library (http://www.cochrane.org/index.htm), including Cochrane database for Systematic Reviews, Database of Abstracts of Reviews of Effectiveness, Cochrane Controlled Trials Register and Cochrane Review Methodology Database; Web of Science (http://www.isiknowledge.com), Controlled-Trial Database (http://controlled-trial.com), Clinical Trials-US National Institutes of Health (http://www.clinicaltrials.gov), the National Institute for Health and Clinical Excellence (http://www. nice.org.uk) and the Virtual Health Library (Bireme-Latin America; www.bireme.br). No restrictions were placed on the language or year of publication. Searches were performed in July 2010, and a new search was conducted in August 2012 in Medline to update the findings.

The following search strategy was used in the Medline: caries OR Dental Caries (Mesh) OR dental decay OR DMF index (Mesh) OR decayed teeth OR DMFS OR DMFT AND socioeconomic factors (Mesh) OR social class (Mesh) OR educational status (Mesh) OR educational level OR socioeconomic condition OR socioeconomic level OR socioeconomic determinant* OR social determinant* OR income (Mesh) OR poverty (Mesh) OR risk factors (Mesh) OR occupational class. In Medline, the search was limited to include only studies with subjects $\geq 19$ years of age.

The following search strategy was used for Cochrane Library and Web of Science: caries OR Dental Caries (Mesh) OR dental decay OR DMF index (Mesh) OR decayed teeth) AND (socioeconomic factors (Mesh) OR social class (Mesh) OR educational status (Mesh) OR educational level OR socioeconomic condition OR socioeconomic level OR socioeconomic determinant* OR social determinant* OR income (Mesh) OR poverty (Mesh) OR risk factors (Mesh).

The Virtual Health Library (Bireme) included both the Latin-American and Caribbean System on Health Science Information (Lilacs) and Brazilian Library of Dentistry (BBO) databases. In the Lilacs, BBO and Clinical Trials databases, two keywords were used at a time because these databases did not support the entire search strategy. In the Lilacs and BBO databases, keywords in Portuguese were also used. In the Controlled-Trial and National Institute for Health and Clinical Excellence databases, one keyword was used at a time.

The search was conducted by three researchers (Simone Melo Costa, Maria de Lourdes Carvalho Bonfim and Carolina de Castro Martins). The studies were entered into the Reference Manager ${ }^{\circledR}$ programs, and a list was generated for analysis and selection.

\subsection{Selection of Studies and Data Extraction}

Studies retrieved from the databases were selected after reading the abstracts and titles, following a calibration exercise with $10 \%$ of the studies read by three independent reviewers to determine interexaminer agreement (Kappa: 0.68 to 0.97 ). Disagreements were resolved by consensus. The following were the inclusion criteria for the initial selection process: reviews, epidemiological studies with subjects between 19 and 60 years of age, studies addressing risk factors for dental caries and studies reporting socioeconomic indicators. No clinical trials were found by the searches although all caution was taken to try to find them. For this reason, no clinical trials are considered this in this review. Reviews were included, and their reference lists were searched in turn for any studies not retrieved by 
the electronic search. However, this process yielded no further studies. Initially, all studies addressing risk factors for dental caries were selected, even if socioeconomic indicators were not the main subject. This was a try to find hidden studies that reported socioeconomic indicators as confounders but in which they was not mentioned in the title nor in the abstract. In this fashion, more studies were selected for full text analysis than were expected to meet the review conditions and it was an effort to find important related studies.

The exclusion criteria are presented in Figure 1. Studies involving patients younger than 19 or older than 60 years were excluded; studies involving other outcomes (dental fluorosis, periodontal disease and others); studies evaluating fluorides, xylitol or educational programs; case reports; studies reporting oral health related quality of life as main outcome; laboratorial and diagnostic studies (X ray or clinical examination); studies evaluating treatment needs, health services and satisfaction with dental services; studies related to diet and nutrition; studies that did not perform clinical examination to evaluate dental caries; studies reporting risk factors related to alcohol, tobacco and drugs use; studies of validation methods, development of new methods or indexes; studies that selected groups based on demographic location (rural and urban or ethnic groups); studies without statistical analysis (only frequency data); mortality groups or high specific groups as diabetics, pregnancy and others; and reviews not related to dental caries.

The full texts were obtained from the selected studies. Seventeen authors were contacted by email, and eleven authors answered these emails [10-20]. Among the 189 studies, the full texts for only six could not be found [21-26]. The full texts of the studies were read by two independent reviewers (Simone de Melo Costa and Maria de Lourdes Carvalho Bonfim) following a calibration exercise with 10 studies. Disagreements were resolved by consensus. In this phase, reviews and studies that did not report statistical tests for dental caries and socioeconomic indicators (i.e., studies that reported only prevalence data or descriptive data) and studies with populations involving individuals younger than 19 years or older than 60 years were excluded. Data extraction was conducted by two independent reviewers (Simone de Melo Costa and Maria de Lourdes Carvalho Bonfim). It was developed a form for data extraction and all studies were evaluated considering this form.

\subsection{Quality Assessment}

Two independent reviewers (Simone de Melo Costa and Maria de Lourdes Carvalho Bonfim) evaluated the quality of the studies using the Newcastle-Ottawa scale for cohort studies. Cross-sectional studies were evaluated using the Newcastle-Ottawa scale modified for case-control studies [27]. Study quality was rated on a scale from 1 (very poor) to 9 (high). Disagreements were resolved by consensus. The included ecological study was evaluated as a cross-sectional study.

\subsection{Data Synthesis}

The extraction of the data was based on the study design, population characteristics, the measures used for dental caries and the type of socioeconomic indicators used. A high degree of heterogeneity was found among the methodologies and types of socioeconomic indicators used. Dental caries and socioeconomic variables had several categories and cut points. Therefore, it was not possible to group data for meta-analysis. Instead, narrative synthesis was conducted in this review. 
Figure 1. Flowchart of study selection.

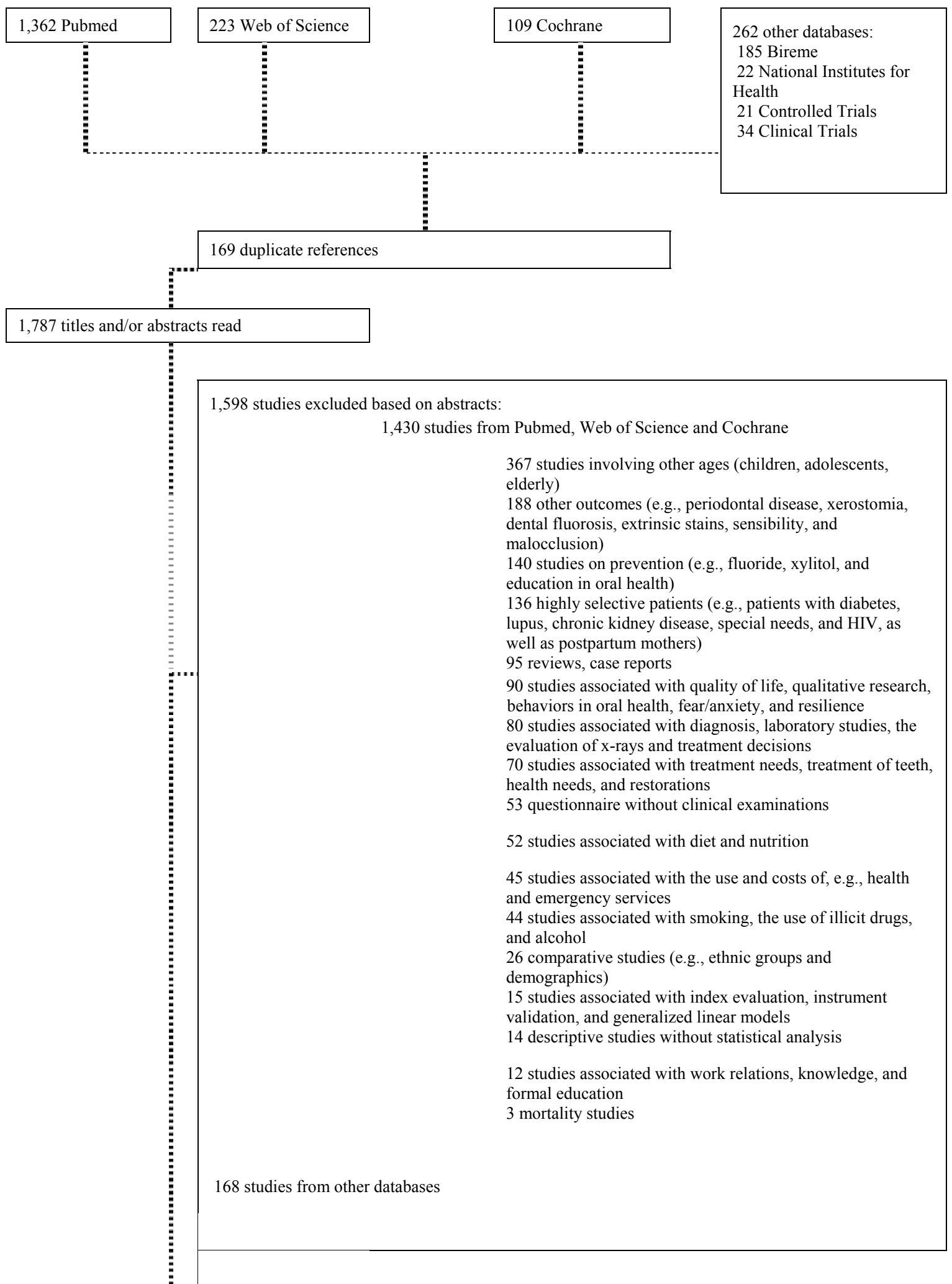


Figure 1. Cont.

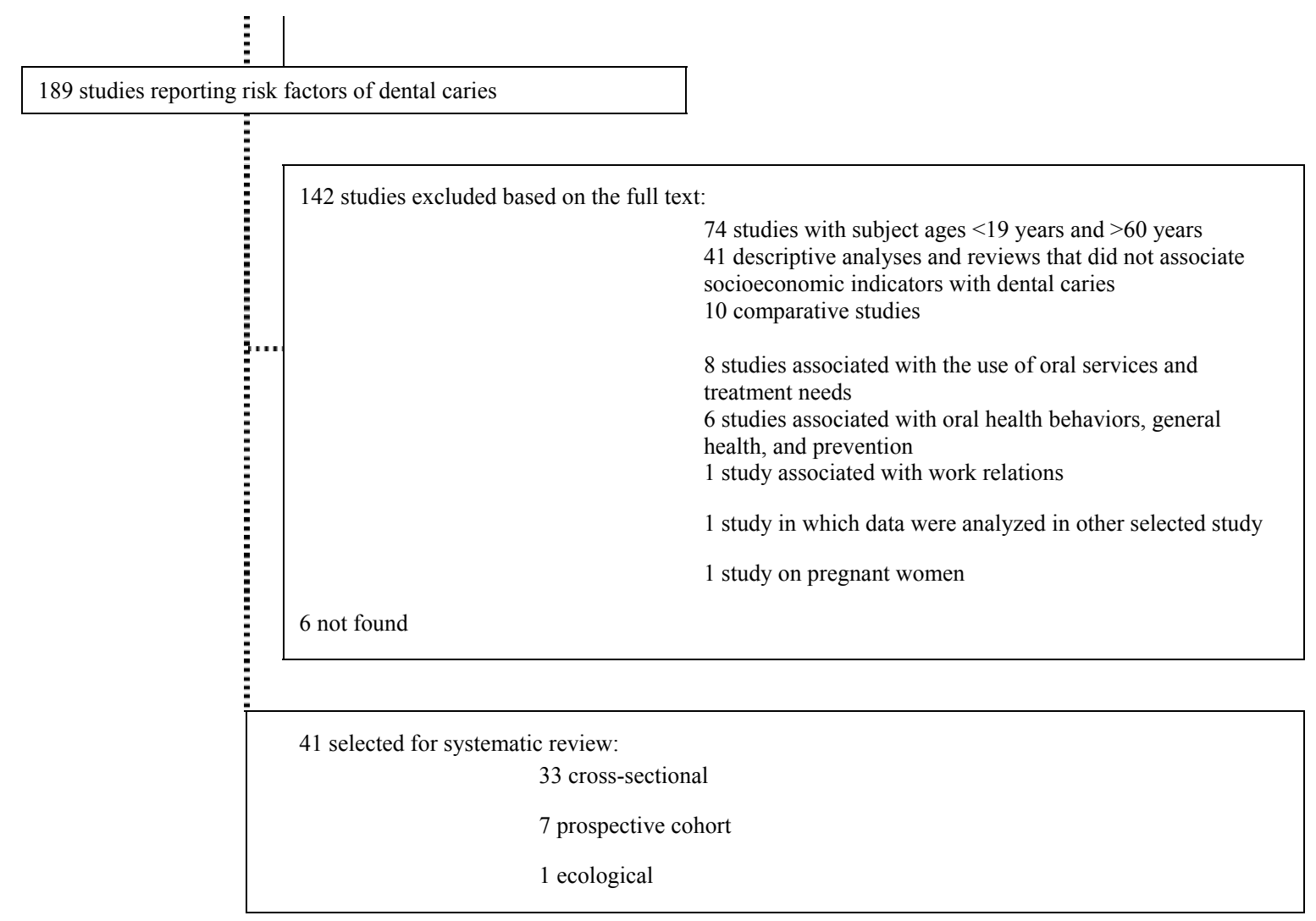

\section{Results}

A total of 1,957 potentially relevant records were found in the seven databases, 169 of which were duplicated. Thus, the abstracts of 1,787 studies were read. A total of 1,598 references were excluded based on the abstracts, and 189 were selected for full-text analysis, 41 of which were selected for inclusion. The systematic review comprised 33 cross-sectional studies, seven prospective cohort studies and one ecological study (Figure 1). No clinical trials were found that evaluated the association between dental caries and socioeconomic indicators.

\subsection{Quality Assessment}

Agreement between the reviewers on each item of the Newcastle-Ottawa scale was $100 \%$. The characteristics of the studies are displayed in Tables 1-3. Regarding quality, cross-sectional studies received between five and eight points [12,16-18,28-56] (Table 1), the ecological study received five points [57], one prospective cohort study received the highest score of nine points [58] and three prospective cohort studies received eight points each [59-61]. The studies by Bille [62] and Holst and Schuller [63] received six points and Hahn et al. [64] received seven points. Although these were originally cohort studies, the results of the socioeconomic data were derived from a cross-sectional presentation (Table 2). 
Table 1. Study characteristics and results reported from cross-sectional studies.

\begin{tabular}{|c|c|c|c|c|c|c|c|c|c|}
\hline Authors, year & Country & $\begin{array}{l}\text { Location of } \\
\text { data } \\
\text { collection }\end{array}$ & $\begin{array}{c}\text { Total } \\
\text { number of } \\
\text { respondents } \\
\text { (Final } \\
\text { sample size) } \\
\end{array}$ & $\begin{array}{l}\text { Subjects' } \\
\text { age } \\
\text { (years) }\end{array}$ & Caries index & Socioeconomic indicator & Other measures & $\begin{array}{c}\text { Association between } \\
\text { socioeconomic indicators } \\
\text { and dental caries }\end{array}$ & $\begin{array}{c}\text { Newcastle- } \\
\text { Ottawa } \\
\text { scale }\end{array}$ \\
\hline $\begin{array}{l}\text { Nikias et al., } \\
1975 \text { [28] }\end{array}$ & USA & $\begin{array}{l}\text { Clinical } \\
\text { setting }\end{array}$ & $\begin{array}{c}1,290 \\
(1,122)\end{array}$ & $19+$ years & $\begin{array}{c}\text { Decayed teeth } \\
\text { Mean } \\
\text { Number of } \\
\text { decayed teeth: } \\
\text { none, } 1-2,3 \text { or } \\
\text { more }\end{array}$ & $\begin{array}{l}\text { Status: poverty and non- } \\
\text { poverty }\end{array}$ & $\begin{array}{l}\text { Gender, age, } \\
\text { edentulousness, } \\
\text { soft tissue lesions, } \\
\text { gingival status, } \\
\text { oral hygiene } \\
\text { levels, visit to } \\
\text { dentist, frequency } \\
\text { of brushing }\end{array}$ & $\begin{array}{c}\text { Poverty and more dental } \\
\text { caries } \\
(p<0.05) *\end{array}$ & $5(9)$ \\
\hline $\begin{array}{c}\text { Hansen, } 1977 \\
\text { [29] }\end{array}$ & Norway & $\begin{array}{l}\text { Clinical } \\
\text { setting }\end{array}$ & 177 (117) & 35 years & $\begin{array}{c}\text { DMFT } \\
\text { Decayed teeth } \\
\text { Mean }\end{array}$ & $\begin{array}{l}\text { Years of schooling } \\
(\leq 10 \text { and }>10)\end{array}$ & Gender & $\begin{array}{c}\text { Less schooling and more } \\
\text { dental caries } \\
(p>0.05)^{*}\end{array}$ & $6(9)$ \\
\hline $\begin{array}{c}\text { Meyer et al., } \\
1983[30]\end{array}$ & Portugal & $\begin{array}{l}\text { Clinical } \\
\text { setting }\end{array}$ & $73(73)$ & $\begin{array}{c}21 \text { to } \\
30 \text { years }\end{array}$ & $\begin{array}{l}\text { DMFT } \\
\text { DMFS } \\
\text { Mean }\end{array}$ & $\begin{array}{l}\text { Lower socioeconomic } \\
\text { status (manual laborers) } \\
\text { and higher socioeconomic } \\
\text { status (the first three } \\
\text { classes of students } \\
\text { graduating from the new } \\
\text { dental school in Lisbon) }\end{array}$ & $\begin{array}{l}\text { Periodontal Index, } \\
\text { gender, oral } \\
\text { hygiene }\end{array}$ & $\begin{array}{l}\text { Lower socioeconomic } \\
\text { status and lower DMFT } \\
=15.9 \pm 6.3,10.0 \pm 5.0 \\
\text { DMFS }=42.6 \pm 25.0 \\
24.1 \pm 15.3 \\
(p<0.05)^{*}\end{array}$ & $6(9)$ \\
\hline $\begin{array}{c}\text { Tervonen et al., } \\
1991 \text { [31] }\end{array}$ & Finland & Not reported & $1,600(883)$ & $\begin{array}{c}25,35, \\
50 \text { years }\end{array}$ & $\begin{array}{c}\text { Number of } \\
\text { decayed teeth } \\
(\mathrm{D}<7 \text { or } \geq 7)\end{array}$ & $\begin{array}{l}\text { Years of education: } \\
\text { university level, college, } \\
\text { vocational school, } \\
\text { compulsory, secondary } \\
\text { school, less than } 6 \text { years } \\
\text { of junior high school. }\end{array}$ & $\begin{array}{l}\text { Periodontal } \\
\text { disease, age, } \\
\text { gender, number of } \\
\text { teeth, regularity } \\
\text { of attendance for } \\
\text { treatment, attitude } \\
\text { to preservation of } \\
\text { teeth, and others. }\end{array}$ & $\begin{array}{l}\text { Less schooling and more } \\
\text { decayed teeth } \\
(p<0.05) * * \\
\text { OR }=1.12(1.03-1.20)\end{array}$ & $7(9)$ \\
\hline
\end{tabular}


Table 1. Cont

\begin{tabular}{|c|c|c|c|c|c|c|c|c|c|}
\hline Authors, year & Country & $\begin{array}{l}\text { Location of } \\
\text { data } \\
\text { collection }\end{array}$ & $\begin{array}{c}\text { Total } \\
\text { number of } \\
\text { respondents } \\
\text { (Final } \\
\text { sample size) } \\
\end{array}$ & $\begin{array}{l}\text { Subjects' } \\
\text { age } \\
\text { (years) }\end{array}$ & Caries index & Socioeconomic indicator & Other measures & $\begin{array}{c}\text { Association between } \\
\text { socioeconomic indicators } \\
\text { and dental caries }\end{array}$ & $\begin{array}{c}\text { Newcastle- } \\
\text { Ottawa } \\
\text { scale }\end{array}$ \\
\hline $\begin{array}{c}\text { Marcenes \& } \\
\text { Sheiham, } 1992 \\
\text { [32] }\end{array}$ & Brazil & $\begin{array}{c}\text { Subjects' } \\
\text { homes }\end{array}$ & $164(164)$ & $\begin{array}{l}35 \text { to } \\
44 \text { years }\end{array}$ & $\begin{array}{l}\text { DMFS } \\
\text { Mean }\end{array}$ & $\begin{array}{c}\text { Socioeconomic status by } \\
\text { ABA-ABIPEME }\end{array}$ & $\begin{array}{c}\text { Periodontal status, } \\
\text { age, frequency of } \\
\text { brushing, sugar } \\
\text { consumption, } \\
\text { frequency of } \\
\text { dental care, } \\
\text { mental demand of } \\
\text { work, marital } \\
\text { quality, and } \\
\text { others }\end{array}$ & $\begin{array}{l}\text { Lower socioeconomic } \\
\text { status and greater DMFS } \\
\qquad(p<0.05) * \\
(p>0.05) * *\end{array}$ & $7(9)$ \\
\hline $\begin{array}{c}\text { Eriksen et al., } \\
1996 \text { [33] }\end{array}$ & Portugal & $\begin{array}{l}\text { Clinical } \\
\text { setting }\end{array}$ & $322(196)$ & $\begin{array}{c}30 \text { to } \\
39 \text { years }\end{array}$ & $\begin{array}{c}\text { Number of } \\
\text { decayed surfaces- } \\
\text { DS } \\
\text { Mean }\end{array}$ & $\begin{array}{l}\text { Social class: class } 1 \text {, } \\
\text { class } 2 \text { and class } 3 \\
\text { Years of schooling: } \\
\quad \leq 10 \text { and }>10\end{array}$ & $\begin{array}{c}\text { Smoking, } \\
\text { psychological } \\
\text { status, eating } \\
\text { between meals, } \\
\text { tooth cleaning } \\
\text { (OHI-S), brushing } \\
\text { frequency, } \\
\text { interdental } \\
\text { cleaning, use of } \\
\text { fluoridated } \\
\text { toothpaste, } \\
\text { gender, regular } \\
\text { dental visits, and } \\
\text { others }\end{array}$ & $\begin{array}{l}\text { Lower social class and } \\
\text { more decayed surfaces } \\
\qquad(p>0.05)^{*} \\
\text { DS }=12.6 \pm 11.1 \\
12.4 \pm 11.6,15.7 \pm 13.2 \\
\text { Less schooling and more } \\
\text { decayed surfaces } \\
(p>0.05) * \\
\text { DS }=14.5 \pm 12.7 \\
11.7 \pm 10.8\end{array}$ & $6(9)$ \\
\hline
\end{tabular}


Table 1. Cont

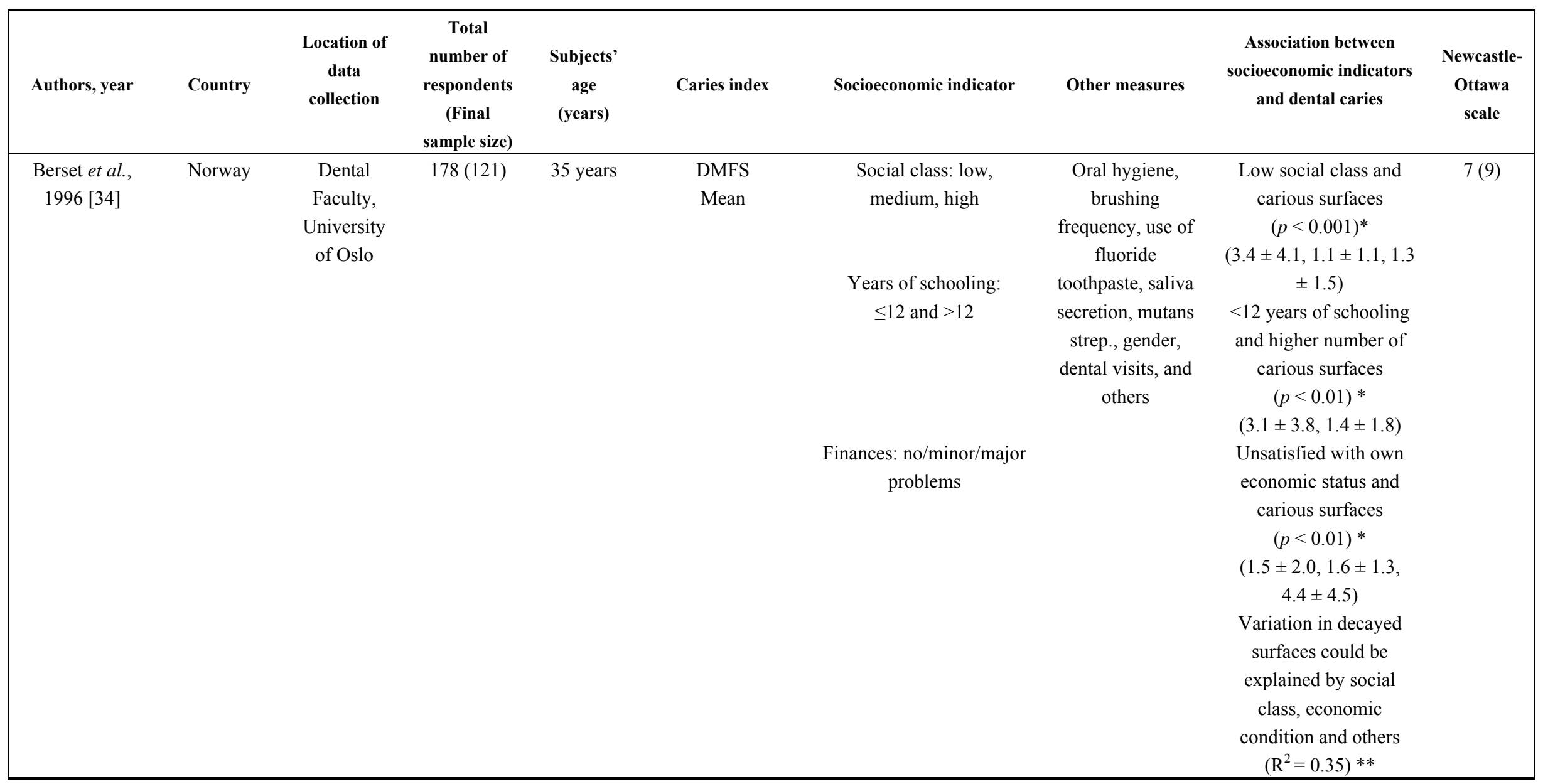


Table 1. Cont.

\begin{tabular}{|c|c|c|c|c|c|c|c|c|c|}
\hline Authors, year & Country & $\begin{array}{l}\text { Location of } \\
\text { data } \\
\text { collection }\end{array}$ & $\begin{array}{c}\text { Total } \\
\text { number of } \\
\text { respondents } \\
\text { (Final } \\
\text { sample size) }\end{array}$ & $\begin{array}{l}\text { Subjects' } \\
\text { age } \\
\text { (years) }\end{array}$ & Caries index & Socioeconomic indicator & Other measures & $\begin{array}{c}\text { Association between } \\
\text { socioeconomic indicators } \\
\text { and dental caries }\end{array}$ & $\begin{array}{c}\text { Newcastle- } \\
\text { Ottawa } \\
\text { scale }\end{array}$ \\
\hline $\begin{array}{c}\text { Hescot et al., } \\
1997 \text { [35] }\end{array}$ & France & $\begin{array}{l}\text { Exam } \\
\text { carried out } \\
\text { on clusters } \\
\text { (transport- } \\
\text { able dental } \\
\text { chair) }\end{array}$ & $\begin{array}{c}1,000 \\
(1,000)\end{array}$ & $\begin{array}{c}35 \text { to } \\
44 \text { years }\end{array}$ & $\begin{array}{l}\text { DMFT and } \\
\text { decayed teeth } \\
\text { (DT) } \\
\text { Mean }\end{array}$ & $\begin{array}{l}\text { Occupational group: high, } \\
\text { medium, low (derived } \\
\text { from the combination of } \\
\text { occupational activity, } \\
\text { educational level and } \\
\text { household income) }\end{array}$ & $\begin{array}{l}\text { Gender, residence } \\
\text { (urban, rural), one } \\
\text { or more surface } \\
\text { fillings, treatment } \\
\text { need (pulp care, } \\
\text { extraction or other } \\
\text { treatment) }\end{array}$ & $\begin{array}{c}\text { Lower occupational } \\
\text { group and more decayed } \\
\text { teeth } \\
(p>0.05) * \\
\mathrm{DT}=0.9 \pm 1.8 \\
1.2 \pm 2.2,1.3 \pm 2.0\end{array}$ & $6(9)$ \\
\hline $\begin{array}{c}\text { Schuller, } 1999 \\
{[36]}\end{array}$ & Norway & Not reported & $\begin{array}{c}\text { Evaluation } \\
\text { of two } \\
\text { sample } \\
\text { groups in } \\
1983 \\
(945(796)) \\
\text { and } 1994 \\
(702(454))\end{array}$ & $\begin{array}{c}23 \text { to } \\
24 \text { years }\end{array}$ & $\begin{array}{l}\text { Decayed and } \\
\text { filled surfaces } \\
\text { (DFS) } \\
\text { Mean }\end{array}$ & $\begin{array}{l}\text { Years of education: } \\
\quad \leq 12 \text { and } \geq 13\end{array}$ & $\begin{array}{l}\text { Gender, residence } \\
\text { (urban, rural), } \\
\text { time since last } \\
\text { dental visit, type } \\
\text { of dental clinic, } \\
\text { use of dental } \\
\text { service, treatment } \\
\text { received, oral } \\
\text { hygiene score, } \\
\text { and others }\end{array}$ & $\begin{array}{c}\text { Less schooling and more } \\
\text { decayed and filled } \\
\text { surfaces in both years } \\
\text { analyzed } \\
(p<0.05) * \\
(1983=40.7,37.0) \\
(1994=22.3,15.6)\end{array}$ & $6(9)$ \\
\hline $\begin{array}{c}\text { Sgan-Cohen et } \\
\text { al., } 1999 \text { [37] }\end{array}$ & Israel & $\begin{array}{l}\text { Clinical } \\
\text { setting of } \\
\text { military } \\
\text { institute }\end{array}$ & $\begin{array}{c}1,300 \\
(1,084)\end{array}$ & $\begin{array}{c}25 \text { to } \\
44 \text { years }\end{array}$ & $\begin{array}{c}\text { DMFT } \\
\text { Decayed teeth } \\
\text { (DT) } \\
\text { Mean }\end{array}$ & $\begin{array}{l}\text { Years of education: } \\
<12,12 \text { and }>12\end{array}$ & Gender, age & $\begin{array}{l}\text { Less schooling and more } \\
\text { decayed teeth } \\
\quad(p<0.001)^{*} \\
\mathrm{DT}=1.75 \pm 2.4 \\
1.53 \pm 2.2,0,89 \pm 1.4 \\
\quad(p<0.001) * * \\
\text { Number of years of } \\
\text { education with the D } \\
\text { component- } \\
\mathrm{Rb}=-0.16\end{array}$ & $6(9)$ \\
\hline
\end{tabular}


Table 1. Cont.

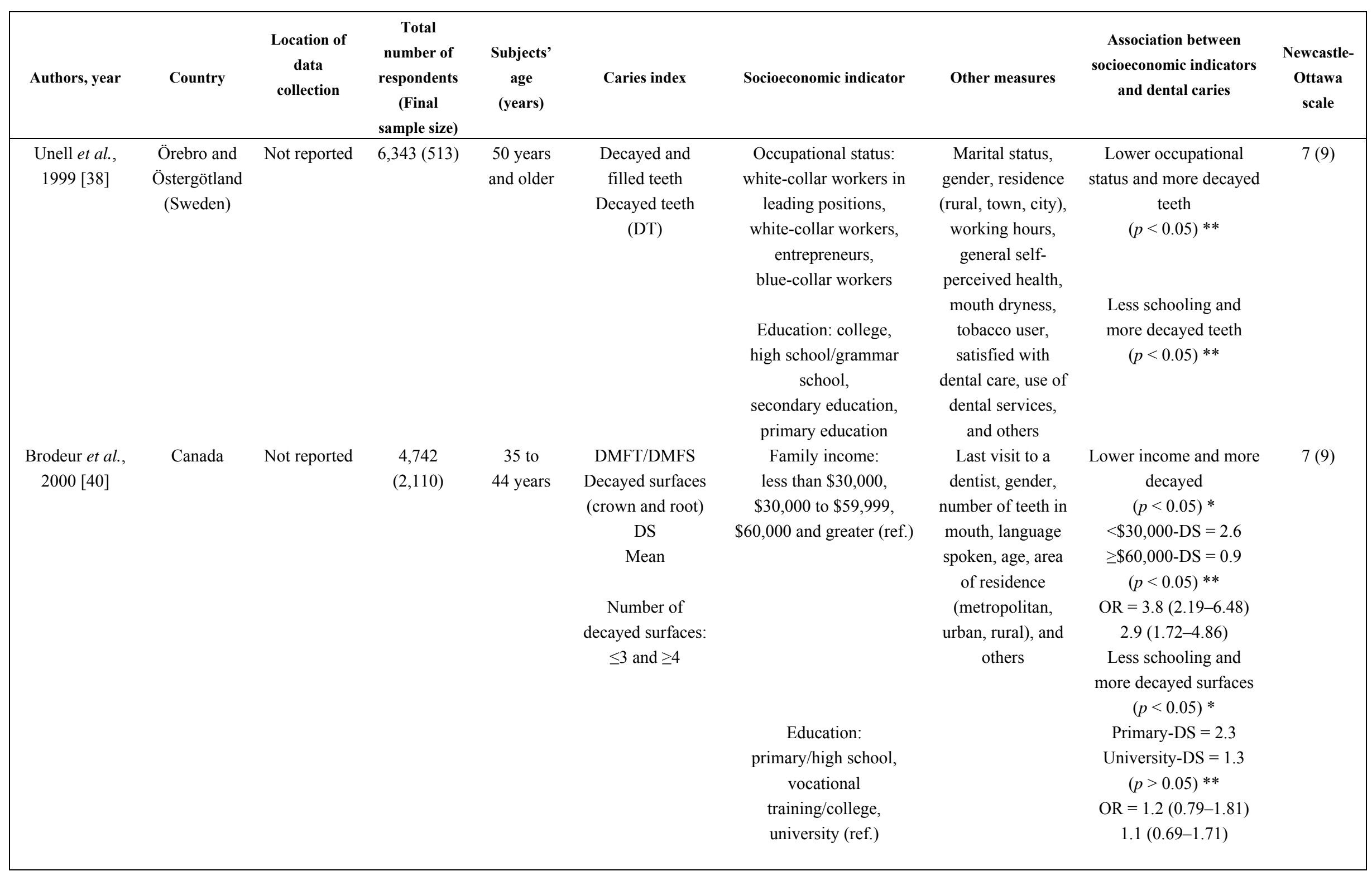


Table 1. Cont

\begin{tabular}{|c|c|c|c|c|c|c|c|c|c|}
\hline Authors, year & Country & $\begin{array}{l}\text { Location of } \\
\text { data } \\
\text { collection }\end{array}$ & $\begin{array}{c}\text { Total } \\
\text { number of } \\
\text { respondents } \\
\text { (Final } \\
\text { sample size) }\end{array}$ & $\begin{array}{l}\text { Subjects' } \\
\text { age } \\
\text { (years) }\end{array}$ & Caries index & Socioeconomic indicator & Other measures & $\begin{array}{c}\text { Association between } \\
\text { socioeconomic indicators } \\
\text { and dental caries }\end{array}$ & $\begin{array}{c}\text { Newcastle- } \\
\text { Ottawa } \\
\text { scale }\end{array}$ \\
\hline $\begin{array}{l}\text { Doughan et al., } \\
2000[41]\end{array}$ & Lebanon & $\begin{array}{l}\text { Clinical } \\
\text { setting }\end{array}$ & $401(401)$ & $\begin{array}{c}35 \text { to } \\
44 \text { years }\end{array}$ & $\begin{array}{c}\text { DMFT } \\
\text { Decayed teeth- } \\
\text { DT } \\
\text { Mean }\end{array}$ & $\begin{array}{l}\text { Socioeconomic status: } \\
\text { low, middle, high }\end{array}$ & $\begin{array}{l}\text { Gender, residence } \\
\text { (urban, rural), } \\
\text { treatment need }\end{array}$ & $\begin{array}{c}\text { Worse socioeconomic } \\
\text { status and more decayed } \\
\text { teeth } \\
(p<0.05) * \\
\mathrm{DT}=5.7 \pm 5.7,4.0 \pm \\
4.7,2.2 \pm 2.8)\end{array}$ & $7(9)$ \\
\hline $\begin{array}{l}\text { Skudutyte et } \\
\text { al., } 2000 \text { [42] }\end{array}$ & Lithuania & $\begin{array}{l}\text { Clinical } \\
\text { setting }\end{array}$ & $767(380)$ & $\begin{array}{c}35 \text { to } \\
44 \text { years }\end{array}$ & $\begin{array}{c}\text { DMFT/DMFS } \\
\text { Decayed teeth-DT } \\
\text { Decayed surfaces } \\
\text { Mean/Median }\end{array}$ & $\begin{array}{l}\text { Education: low, medium, } \\
\text { high }\end{array}$ & $\begin{array}{l}\text { Gender, residence } \\
\text { (urban, rural), } \\
\text { fluoridated water, } \\
\text { oral hygiene } \\
\text { index (OHI-S) }\end{array}$ & $\begin{array}{l}\text { Less schooling and more } \\
\text { decayed teeth } \\
(p<0.05) * \\
\text { DT }=3.0,2.0,1.0\end{array}$ & $6(9)$ \\
\hline $\begin{array}{l}\text { Paulander et } \\
\text { al., } 2003 \text { [45] }\end{array}$ & Sweden & $\begin{array}{l}\text { Clinical } \\
\text { setting }\end{array}$ & $588(588)$ & $\begin{array}{c}35 \text { and } \\
50 \text { years }\end{array}$ & $\begin{array}{c}\text { DMFS } \\
\text { Decayed surfaces- } \\
\text { DS } \\
\text { Mean }\end{array}$ & Education: low and high & $\begin{array}{l}\text { Number of teeth, } \\
\text { probing } \\
\text { attachment level, } \\
\text { periodontal } \\
\text { treatment needs, } \\
\text { prevalence of } \\
\text { dental caries, } \\
\text { dietary habits, and } \\
\text { others }\end{array}$ & $\begin{array}{c}\text { Less schooling and more } \\
\text { DS } \\
(p>0.05)^{*} \\
35 \text { years- } \\
\text { DS }=1.3(-0.2-2.7) \\
0.5(-0.3-1.1) \\
50 \text { years- } \\
\text { DS }=0.4(0.2-0.6) \\
0.4(0.1-0.7)\end{array}$ & $6(9)$ \\
\hline
\end{tabular}


Table 1. Cont

\begin{tabular}{|c|c|c|c|c|c|c|c|c|c|}
\hline Authors, year & Country & $\begin{array}{l}\text { Location of } \\
\text { data } \\
\text { collection }\end{array}$ & $\begin{array}{l}\text { Total } \\
\text { number of } \\
\text { respondents } \\
\text { (Final } \\
\text { sample size) }\end{array}$ & $\begin{array}{l}\text { Subjects' } \\
\text { age } \\
\text { (years) }\end{array}$ & Caries index & Socioeconomic indicator & Other measures & $\begin{array}{c}\text { Association between } \\
\text { socioeconomic indicators } \\
\text { and dental caries }\end{array}$ & $\begin{array}{c}\text { Newcastle- } \\
\text { Ottawa } \\
\text { scale }\end{array}$ \\
\hline $\begin{array}{c}\text { Senna et al., } \\
2005 \text { [47] }\end{array}$ & Italy & $\begin{array}{l}\text { Clinical } \\
\text { setting of a } \\
\text { military } \\
\text { institute }\end{array}$ & $\begin{array}{c}3,661 \\
(2,908)\end{array}$ & $\begin{array}{c}19 \text { to } \\
25 \text { years }\end{array}$ & $\begin{array}{c}\text { DMFT } \\
\text { Decayed teeth-DT } \\
\text { Mean }\end{array}$ & $\begin{array}{l}\text { Educational level: } \\
\text { completed college or } \\
\text { graduate school, high } \\
\text { school, secondary school } \\
\text { and primary school }\end{array}$ & $\begin{array}{c}\text { Gingival and } \\
\text { periodontal status }\end{array}$ & $\begin{array}{c}\text { Less schooling and more } \\
\begin{array}{c}\text { decayed teeth } \\
(p<0.05) * \\
\text { DT }=0.7 \pm 1.2 \\
1.0 \pm 1.4,1.5 \pm 1.9 \\
1.9 \pm 1.9\end{array}\end{array}$ & $5(9)$ \\
\hline $\begin{array}{c}\text { Badel et al., } \\
2006 \text { [12] }\end{array}$ & Croatia & $\begin{array}{l}\text { Clinical } \\
\text { setting of a } \\
\text { military } \\
\text { institute }\end{array}$ & $248(248)$ & $\begin{array}{c}19 \text { to } \\
29 \text { years }\end{array}$ & $\begin{array}{c}\text { DMFT } \\
\text { Decayed teeth } \\
\text { (quartile cutoffs: } \\
\text { Q25, Q50 and } \\
\text { Q75\%) }\end{array}$ & $\begin{array}{c}\text { Schooling in } 3 \text { categories: } \\
\text { primary, secondary, } \\
\text { university }\end{array}$ & Sugar intake & $\begin{array}{l}\text { Less schooling and more } \\
\text { decayed teeth } \\
(p<0.05) * \\
\text { Q75\% }=5,4,1.5\end{array}$ & $6(9)$ \\
\hline $\begin{array}{c}\text { Julihn et al., } \\
2006 \text { [48] }\end{array}$ & Sweden & $\begin{array}{l}\text { Clinical } \\
\text { setting }\end{array}$ & $800(696)$ & 19 years & $\begin{array}{c}\text { DMFS } \\
<10 \text { and } \geq 10\end{array}$ & $\begin{array}{l}\text { Educational level of } \\
\text { father: } \leq 9 \text { years, } 10- \\
12 \text { years, }>12 \text { years } \\
\text { Occupational status of } \\
\text { mother and father: } \\
\text { unemployed, laborer, } \\
\text { white-collar worker }\end{array}$ & $\begin{array}{l}\text { Gender, chronic } \\
\text { disease, country } \\
\text { of birth, years } \\
\text { living in Sweden, } \\
\text { oral hygiene, } \\
\text { attitude toward } \\
\text { dental care, dental } \\
\text { fear, gingival } \\
\text { bleeding index } \\
\text { (GBI), and others }\end{array}$ & $\begin{array}{l}\text { Less education of the } \\
\text { father and more decayed } \\
\text { surfaces } \\
\qquad(p<0.01)^{*} \\
(p>0.05)^{* *} \\
\text { Worse occupation of the } \\
\text { father and more decayed } \\
\text { surfaces } \\
(p<0.01)^{*} \\
(p>0.05) * * \\
\text { Worse occupation of the } \\
\text { mother and more } \\
\text { decayed surfaces } \\
(p<0.01)^{*} \\
(p>0.05) * *\end{array}$ & $7(9)$ \\
\hline
\end{tabular}


Table 1. Cont

\begin{tabular}{|c|c|c|c|c|c|c|c|c|c|}
\hline Authors, year & Country & $\begin{array}{l}\text { Location of } \\
\text { data } \\
\text { collection }\end{array}$ & $\begin{array}{c}\text { Total } \\
\text { number of } \\
\text { respondents } \\
\text { (Final } \\
\text { sample size) }\end{array}$ & $\begin{array}{l}\text { Subjects' } \\
\text { age } \\
\text { (years) }\end{array}$ & Caries index & Socioeconomic indicator & Other measures & $\begin{array}{c}\text { Association between } \\
\text { socioeconomic indicators } \\
\text { and dental caries }\end{array}$ & $\begin{array}{c}\text { Newcastle- } \\
\text { Ottawa } \\
\text { scale }\end{array}$ \\
\hline $\begin{array}{c}\text { Varenne et al., } \\
2006[49]\end{array}$ & $\begin{array}{c}\text { Burkina } \\
\text { Faso }\end{array}$ & $\begin{array}{l}\text { Subjects' } \\
\text { homes }\end{array}$ & $493(493)$ & $\begin{array}{c}35 \text { to } \\
44 \text { years }\end{array}$ & $\begin{array}{c}\text { DMFT } \\
\text { Mean } \\
\text { Absence/presence } \\
\text { of caries }\end{array}$ & $\begin{array}{c}\text { Occupation: shop keeper, } \\
\text { government employee, } \\
\text { smallholder/craftsman, } \\
\text { housewife, } \\
\text { farmer/breeder(ref.) } \\
\text { Educational level: high, } \\
\text { moderate, low }\end{array}$ & $\begin{array}{l}\text { Gender, ethnical } \\
\text { group, dental } \\
\text { visits, use of } \\
\text { chewstick, } \\
\text { consumption of } \\
\text { fresh fruits, } \\
\text { location (urban, } \\
\text { rural), and others }\end{array}$ & $\begin{array}{c}\text { Government employee } \\
\text { and higher DMFT } \\
(p<0.05) * * \\
\text { OR }=5.26 \\
\text { High educational level } \\
\text { and higher CPOD } \\
(p<0.05) * * \\
\text { OR }=2.99\end{array}$ & $7(9)$ \\
\hline $\begin{array}{c}\text { Hessari et al., } \\
2007 \text { [51] }\end{array}$ & Iran & $\begin{array}{l}\text { Non- } \\
\text { specific }\end{array}$ & $\begin{array}{c}8,301 \\
(8,301)\end{array}$ & $\begin{array}{c}35 \text { to } \\
44 \text { years }\end{array}$ & $\begin{array}{c}\text { DMFT } \\
\text { Decayed teeth-DT } \\
\text { Mean }\end{array}$ & $\begin{array}{l}\text { Level of education: } \\
\text { illiterate, low, medium, } \\
\text { high }\end{array}$ & $\begin{array}{l}\text { Gender, place of } \\
\text { residence (urban, } \\
\text { rural) }\end{array}$ & $\begin{array}{c}\text { Less schooling and more } \\
\text { decayed teeth } \\
(p<0.05)^{*} \\
\text { Men-DT }=2.7 \pm 2.7 \\
2.8 \pm 2.6,2.4 \pm 2.7 \\
1.9 \pm 2.4 \\
\text { Women-DT }=2.8 \pm 2.7 \\
2.8 \pm 2.8,1.9 \pm 2.4 \\
1.9 \pm 2.6\end{array}$ & $7(9)$ \\
\hline
\end{tabular}


Table 1. Cont

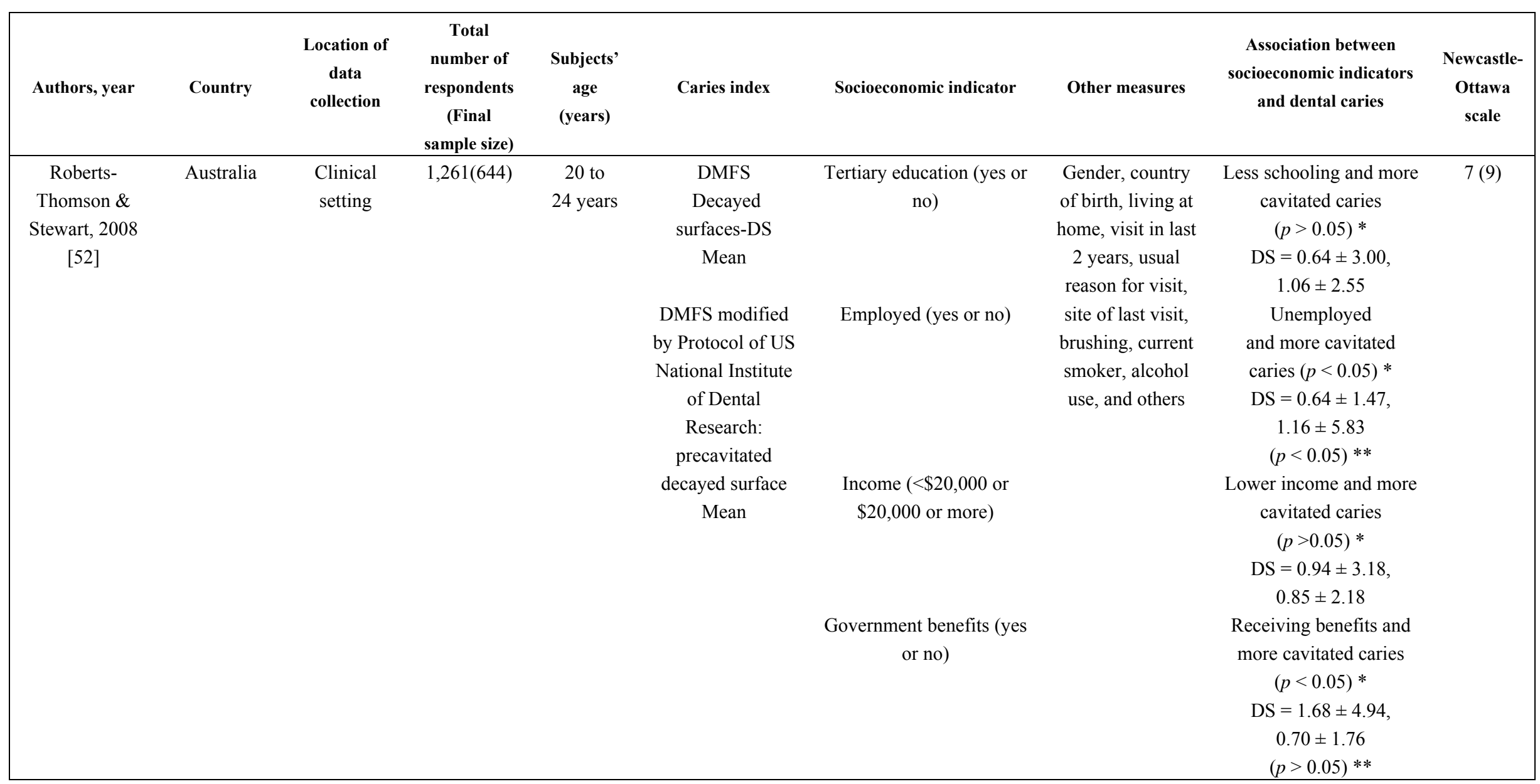


Table 1. Cont

\begin{tabular}{|c|c|c|c|c|c|c|c|c|c|}
\hline Authors, year & Country & $\begin{array}{l}\text { Location of } \\
\text { data } \\
\text { collection }\end{array}$ & $\begin{array}{c}\text { Total } \\
\text { number of } \\
\text { respondents } \\
\text { (Final } \\
\text { sample size) } \\
\end{array}$ & $\begin{array}{c}\text { Subjects' } \\
\text { age } \\
\text { (years) }\end{array}$ & Caries index & Socioeconomic indicator & Other measures & $\begin{array}{c}\text { Association between } \\
\text { socioeconomic indicators } \\
\text { and dental caries }\end{array}$ & $\begin{array}{c}\text { Newcastle- } \\
\text { Ottawa } \\
\text { scale }\end{array}$ \\
\hline $\begin{array}{c}\text { Skudutyte- } \\
\text { Rysstad et al., } \\
2009 \text { [54] }\end{array}$ & Norway & $\begin{array}{c}\text { Clinical } \\
\text { setting }\end{array}$ & 149 (149) & 35 years & $\begin{array}{c}\text { DMFT } \\
\text { DMFS } \\
\text { Decayed surfaces } \\
\text { on dentin ( } \geq 2 \text { and } \\
<2 \text { )-DS }\end{array}$ & $\begin{array}{l}\text { Education: no university } \\
\text { or university } \\
\text { Household income } \\
\text { (NOK/year): } \\
\leq 299,000 \text { (low), } \\
300,000-599,000 \\
\text { (medium) and } \geq 600,000 \\
\text { (high) }\end{array}$ & $\begin{array}{l}\text { Gender, marital } \\
\text { status, region of } \\
\text { birth, dental } \\
\text { anxiety score, } \\
\text { frequency of } \\
\text { brushing, use of } \\
\text { dental floss, } \\
\text { dental visits, time } \\
\text { since last dental } \\
\text { visit, smoking }\end{array}$ & $\begin{array}{l}\text { Less schooling and more } \\
\text { decayed surfaces } \\
\qquad(p<0.05) * \\
\text { Lower income and more } \\
\text { decayed surfaces } \\
(p<0.05) * \\
(p<0.05) * * \\
\text { DS-OR }=4.5(1.9-10.2)\end{array}$ & $6(9)$ \\
\hline $\begin{array}{c}\text { Brennan et al., } \\
2010 \text { [17] }\end{array}$ & Australia & $\begin{array}{l}\text { Clinical } \\
\text { setting }\end{array}$ & 879 (709) & $\begin{array}{c}45 \text { to } \\
54 \text { years }\end{array}$ & $\begin{array}{c}\text { DMFT } \\
\text { Decayed teeth-DT } \\
\text { Mean }\end{array}$ & $\begin{array}{l}\text { Household income: under } \\
\text { AU } \$ 30,000 \text {, AU } \$ 30,000- \\
\$ 60,000 \text {, over AU } \$ 60,000\end{array}$ & $\begin{array}{c}\text { Gender, place of } \\
\text { birth, dental } \\
\text { knowledge of } \\
\text { tooth decay } \\
\text { prevention }\end{array}$ & $\begin{array}{l}\text { Lower income and more } \\
\text { decayed teeth } \\
(p<0.01) * \\
\mathrm{DT}=0.8 \pm 0.13 \\
0.4 \pm 0.07,0.2 \pm 0.03 \\
(p<0.01) * *\end{array}$ & $7(9)$ \\
\hline
\end{tabular}


Table 1. Cont

\begin{tabular}{|c|c|c|c|c|c|c|c|c|c|}
\hline Authors, year & Country & $\begin{array}{l}\text { Location of } \\
\text { data } \\
\text { collection }\end{array}$ & $\begin{array}{c}\text { Total } \\
\text { number of } \\
\text { respondents } \\
\text { (Final } \\
\text { sample size) }\end{array}$ & $\begin{array}{l}\text { Subjects' } \\
\text { age } \\
\text { (years) }\end{array}$ & Caries index & Socioeconomic indicator & Other measures & $\begin{array}{c}\text { Association between } \\
\text { socioeconomic indicators } \\
\text { and dental caries }\end{array}$ & $\begin{array}{c}\text { Newcastle- } \\
\text { Ottawa } \\
\text { scale }\end{array}$ \\
\hline \multirow[t]{2}{*}{$\begin{array}{c}\text { Geyer et al., } \\
2010[16]\end{array}$} & Germany & Not reported & $1,779(925)$ & $\begin{array}{c}35 \text { to } \\
44 \text { years }\end{array}$ & $\begin{array}{c}\text { DMFT } \\
(\leq 21 \text { and }>21)\end{array}$ & $\begin{array}{l}\text { Income in categories: } \\
\text { highest, second highest, } \\
\text { intermediate, second } \\
\text { lowest and lowest; } \\
\text { Educational level: 12- } \\
13 \text { years, } 10 \text { years and 8- } \\
9 \text { years of schooling }\end{array}$ & $\begin{array}{l}\text { Gender, age } \\
\text { (years) }\end{array}$ & $\begin{array}{c}\text { Lower income and } \\
\text { higher DMFT } \\
(p<0.05)^{*} \\
\text { OR }=3.74(1.66-8.46) \\
(p<0.05) * * \\
\text { OR= } 2.34(1.00-5.55) \\
\text { Less schooling and } \\
\text { higher DMFT } \\
(p<0.05) * \\
\text { OR }=3.75(1.99-7.05) \\
(p<0.05) * * \\
\text { OR }=2.95(1.52-5.74)\end{array}$ & $7(9)$ \\
\hline & & & & & & $\begin{array}{c}\text { Cumulative effects: } \\
\text { income + educational } \\
\text { level } 1=\text { highest } \\
\text { socioeconomic positions, } \\
\text { intermediate positions, } \\
\text { lowest positions }\end{array}$ & & $\begin{array}{l}\text { Socioeconomic status - } \\
\text { lowest positions: income } \\
\text { + education level and } \\
\text { higher DMFT } \\
(p<0.05)^{* *} \\
\text { OR }=6.06(2.06-17.87)\end{array}$ & \\
\hline $\begin{array}{c}\text { Celeste et al., } \\
2011 \text { [55] }\end{array}$ & Brazil & $\begin{array}{c}\text { Subjects' } \\
\text { homes }\end{array}$ & $\begin{array}{c}22,839 \\
(20,695)\end{array}$ & $\begin{array}{c}35 \text { to } 44 \\
\text { years }\end{array}$ & $\begin{array}{c}\text { DMFT } \\
\text { Untreated dental } \\
\text { caries } \\
\text { Mean }\end{array}$ & $\begin{array}{l}\text { Gini (quartile), municipal } \\
\text { income (quartile), } \\
\text { household income (based } \\
\text { on minimum wage) }\end{array}$ & $\begin{array}{c}\text { Gender, age, } \\
\text { place of residence } \\
\text { (urban, rural), last } \\
\text { dental visit., } \\
\text { edentulism, } \\
\text { prevalence of } \\
\text { periodontal } \\
\text { attachment loss } \\
>8 \mathrm{~mm}\end{array}$ & $\begin{array}{l}\text { More iniquity results in } \\
\text { more decayed teeth } \\
\quad(p<0.05)^{* *}\end{array}$ & $7(9)$ \\
\hline
\end{tabular}


Table 1. Cont.

\begin{tabular}{|c|c|c|c|c|c|c|c|c|c|}
\hline Authors, year & Country & $\begin{array}{l}\text { Location of } \\
\text { data } \\
\text { collection }\end{array}$ & $\begin{array}{c}\text { Total } \\
\text { number of } \\
\text { respondents } \\
\text { (Final } \\
\text { sample size) } \\
\end{array}$ & $\begin{array}{l}\text { Subjects' } \\
\text { age } \\
\text { (years) }\end{array}$ & Caries index & Socioeconomic indicator & Other measures & $\begin{array}{c}\text { Association between } \\
\text { socioeconomic indicators } \\
\text { and dental caries }\end{array}$ & $\begin{array}{c}\text { Newcastle- } \\
\text { Ottawa } \\
\text { scale }\end{array}$ \\
\hline $\begin{array}{c}\text { Brennan et al., } \\
2011 \text { [18] }\end{array}$ & Australia & $\begin{array}{l}\text { Clinical } \\
\text { setting }\end{array}$ & $879(709)$ & $\begin{array}{c}45 \text { to } \\
54 \text { years }\end{array}$ & $\begin{array}{c}\text { DMFT } \\
\text { Decayed teeth-DT } \\
\text { Mean }\end{array}$ & $\begin{array}{l}\text { Household income (under } \\
\$ 80,000+\text { and }<\$ 80,000 \\
\text { Education: tertiary and } \\
\text { secondary }\end{array}$ & $\begin{array}{l}\text { Dental visit } \\
\text { pattern, dental } \\
\text { self-care (tooth } \\
\text { brushing) }\end{array}$ & $\begin{array}{c}\text { Lower income and more } \\
\text { decayed teeth } \\
(p<0.01) * \\
\text { DT }=0.1 \pm 0.03 \\
0.5 \pm 0.05 \\
(p<0.001) * * \\
\text { Income } \$ 80,000+ \\
\text { Beta }=-0.27 \\
\text { Lesser schooling and } \\
\text { more decayed teeth } \\
(p<0.01) * \\
\text { DT }=0.2 \pm 0.03 \\
0.5 \pm 0.06 \\
(p<0.01) * * \\
\text { Education tertiary Beta } \\
=-0.25\end{array}$ & $7(9)$ \\
\hline $\begin{array}{c}\text { Chandra et al., } \\
2011[56]\end{array}$ & India & Not reported & $\begin{array}{c}1,198 \\
(1,187)\end{array}$ & $\begin{array}{c}19 \text { to } \\
57 \text { years }\end{array}$ & $\begin{array}{c}\text { DMFT } \\
\text { Decayed teeth-DT } \\
\text { Mean }\end{array}$ & $\begin{array}{c}\text { Socioeconomic } \\
\text { status(SES): upper, upper } \\
\text { middle, lower middle, } \\
\text { upper lower, lower } \\
\text { (Modified Kuppuswamy } \\
\text { scale were based on the } \\
\text { 1988-1989) }\end{array}$ & $\begin{array}{c}\text { Gender, } \\
\text { periodontal status, } \\
\text { oral pre- } \\
\text { malignant, } \\
\text { malignant lesions, } \\
\text { demographic } \\
\text { profile, etc. }\end{array}$ & $\begin{array}{c}\text { Lower socioeconomic } \\
\text { status and more decayed } \\
\text { teeth } \\
(p<0.001)^{*} \\
\text { Upper-DT }=0.07 \\
( \pm 0.32) \\
\text { Lower } \\
\text { DT }=0.96( \pm 2.06)\end{array}$ & $6(9)$ \\
\hline
\end{tabular}

* Bivariate analysis; ** multivariate analysis. 
Table 2. Study characteristics and results from seven prospective cohort studies.

\begin{tabular}{|c|c|c|c|c|c|c|c|c|c|}
\hline Authors, year & Country & $\begin{array}{l}\text { Location of } \\
\text { data } \\
\text { collection }\end{array}$ & $\begin{array}{c}\text { Total } \\
\text { number of } \\
\text { respondents } \\
\text { (Final } \\
\text { sample size) }\end{array}$ & $\begin{array}{l}\text { Subjects' } \\
\text { age } \\
\text { (years) }\end{array}$ & Caries index & Socioeconomic indicator & Other measures & $\begin{array}{l}\text { Association between } \\
\text { socioeconomic } \\
\text { indicators and dental } \\
\text { cariess }\end{array}$ & $\begin{array}{l}\text { Newcastle- } \\
\text { Ottawa scale }\end{array}$ \\
\hline $\begin{array}{c}\text { Bille, } 1980 \\
{[62]}\end{array}$ & Denmark & $\begin{array}{l}\text { Subjects' } \\
\text { homes }\end{array}$ & $389(313)$ & $\begin{array}{c}\text { Data } \\
\text { evaluated } \\
\text { at } 20 \\
\text { years of } \\
\text { age } \\
\text { (cross- } \\
\text { sectional) }\end{array}$ & $\begin{array}{l}\text { DMFS } \\
\text { Mean }\end{array}$ & $\begin{array}{l}\text { Subjects' socioeconomic } \\
\text { status and parents' } \\
\text { socioeconomic status by } \\
\text { occupation: } \\
\text { low (unemployment, } \\
\text { unskilled and semiskilled } \\
\text { occupations); } \\
\text { medium (non-manual and } \\
\text { manual skilled } \\
\text { occupations); and } \\
\text { high (intermediate non- } \\
\text { manual, administrative } \\
\text { and professional } \\
\text { occupations) }\end{array}$ & $\begin{array}{l}\text { Gender, dental } \\
\text { visits }\end{array}$ & $\begin{array}{l}\text { Lower socioeconomic } \\
\text { status of parents and } \\
\text { higher DMFS } \\
\qquad(p>0.05)^{*} \\
\text { Lower socioeconomic } \\
\text { status of subject and } \\
\text { higher DMFT } \\
(p<0.01)^{*}\end{array}$ & $6(9)$ \\
\hline $\begin{array}{c}\text { Bjertness et al., } \\
1992[60]\end{array}$ & Norway & Not reported & $\begin{array}{c}116(81) \\
\text { Data } \\
\text { evaluated in } \\
1973 \text { and } \\
1988\end{array}$ & $\begin{array}{l}35 \text { and } \\
50 \text { years }\end{array}$ & $\begin{array}{c}\text { Decayed teeth-DT } \\
\text { Mean }\end{array}$ & $\begin{array}{l}\text { Years at school: } \\
\quad \leq 10 \text { and }>10 \\
\text { Social class: class 1, } \\
\text { class 2, class 3; } \\
\text { Satisfaction with own } \\
\text { finances: unsatisfied, } \\
\text { satisfied; }\end{array}$ & $\begin{array}{l}\text { Alcohol, exercise, } \\
\text { smoking, } \\
\text { psychological } \\
\text { status, sugar } \\
\text { between meals, } \\
\text { teeth cleaning, } \\
\text { use of fluoride, } \\
\text { interdental } \\
\text { cleaning, } \\
\text { allergies, } \\
\text { medications, } \\
\text { regular dental } \\
\text { visits, and others }\end{array}$ & $\begin{array}{c}\text { Less schooling and } \\
\text { more decayed teeth } \\
(p>0.05)^{*} \\
\text { DT }=1.27 \pm 0.452 \\
1.22 \pm 0.417 \\
\text { Lower social class } \\
\text { and more decayed } \\
\text { teeth } \\
(p>0.05)^{*} \\
\text { DT }=1.46 \pm 0.522 \\
1.19 \pm 0.398 \\
1.22 \pm 0.428 \\
\text { Dissatisfaction with } \\
\text { finances and more } \\
\text { decayed teeth } \\
(p>0.05)^{*} \\
\text { DT }=1.14 \pm 0.378 \\
1.24 \pm 0.432\end{array}$ & $8(9)$ \\
\hline
\end{tabular}


Table 2. Cont

\begin{tabular}{|c|c|c|c|c|c|c|c|c|c|}
\hline Authors, year & Country & $\begin{array}{l}\text { Location of } \\
\text { data } \\
\text { collection }\end{array}$ & $\begin{array}{l}\text { Total } \\
\text { number of } \\
\text { respondents } \\
\text { (Final } \\
\text { sample size) } \\
\end{array}$ & $\begin{array}{l}\text { Subjects' } \\
\text { age } \\
\text { (years) }\end{array}$ & Caries index & Socioeconomic indicator & Other measures & $\begin{array}{c}\text { Association between } \\
\text { socioeconomic } \\
\text { indicators and dental } \\
\text { caries }\end{array}$ & $\begin{array}{l}\text { Newcastle- } \\
\text { Ottawa scale }\end{array}$ \\
\hline $\begin{array}{c}\text { Hahn et al., } \\
1999 \text { [64] }\end{array}$ & Germany & $\begin{array}{l}\text { Clinical } \\
\text { setting }\end{array}$ & $\begin{array}{l}300(298) \\
\text { in the } \\
\text { beginning of } \\
\text { the study } \\
\text { (cross- } \\
\text { sectional) }\end{array}$ & $\begin{array}{c}50 \text { to } \\
60 \text { years }\end{array}$ & $\begin{array}{c}\text { DMFT } \\
\text { Decayed root }\end{array}$ & $\begin{array}{c}\text { Education: low, middle, } \\
\text { high }\end{array}$ & $\begin{array}{l}\text { Gender, low- } \\
\text { sugar nutrition, } \\
\text { use of fluoride, } \\
\text { dental attendance, } \\
\text { reason for last } \\
\text { visit, smoking } \\
\text { habits, and others }\end{array}$ & $\begin{array}{l}\text { Less schooling and } \\
\text { decayed roots } \\
\begin{array}{c}(p>0.05)^{* *} \\
\text { Education- } \\
\mathrm{B}=0.0129\end{array}\end{array}$ & $7(9)$ \\
\hline $\begin{array}{l}\text { Gilbert et al., } \\
2001[59]\end{array}$ & USA & Not reported & $\begin{array}{c}873 \text { (723) } \\
\text { (24 months) }\end{array}$ & $\begin{array}{l}45 \text { years } \\
\text { and older }\end{array}$ & $\begin{array}{l}\text { Decayed or filled } \\
\text { root surface } \\
\text { (new root decay } \\
\text { only; new root } \\
\text { filling(s) only; } \\
\text { both new decay } \\
\text { and new filling(s) } \\
\text { or neither) }\end{array}$ & $\begin{array}{l}\text { High school graduate } \\
\text { (yes, no) } \\
\text { Income } \\
\text { ( able to pay, but with } \\
\text { difficulty or not able to } \\
\text { pay) }\end{array}$ & $\begin{array}{l}\text { Regular dental } \\
\text { visits; flosses } \\
\text { daily or more } \\
\text { often; flosses, but } \\
\text { less than daily; } \\
\text { area of residence } \\
\text { (rural, urban) }\end{array}$ & $\begin{array}{l}\text { Less schooling and } \\
\text { more new caries or } \\
\text { restorations } \\
(p<0.05)^{*} \\
\text { Not able to pay and } \\
\text { more new caries } \\
(p<0.05) * * \\
\text { OR }=2.5\end{array}$ & $8(9)$ \\
\hline $\begin{array}{c}\text { Thomson et al., } \\
2004[58]\end{array}$ & $\begin{array}{c}\text { New } \\
\text { Zealand }\end{array}$ & Not reported & $922(838)$ & $\begin{array}{l}\text { Dental } \\
\text { exam for } \\
\text { caries at } \\
\text { ages } 5 \text { and } \\
26 \text { years }\end{array}$ & $\begin{array}{c}\text { DMFS } \\
\text { Decayed surfaces- } \\
\text { DS } \\
\text { Decayed/filled } \\
\text { surfaces } \\
\text { Loss due to caries } \\
\text { Mean }\end{array}$ & $\begin{array}{l}\text { Socioeconomic trajectory } \\
\text { (evaluated at } 5 \text { and } \\
26 \text { years of age): high- } \\
\text { high, low-high, high-low, } \\
\text { low-low }\end{array}$ & $\begin{array}{c}\text { Tooth loss, } \\
\text { periodontal } \\
\text { disease, self-care, } \\
\text { brush less than } \\
\text { once daily, } \\
\text { gender, time spent } \\
\text { living in } \\
\text { fluoridated area }\end{array}$ & $\begin{array}{l}\text { Low-low and high- } \\
\text { low socioeconomic } \\
\text { trajectories and more } \\
\text { decayed surfaces } \\
(p<0.05) * * \\
\text { Mean DS } \\
\text { High-high }=1.26 \\
\text { Low-high }=1.61 \\
\text { High-low }=1.94 \\
\text { Low-low }=2.05\end{array}$ & $9(9)$ \\
\hline
\end{tabular}


Table 2. Cont

\begin{tabular}{|c|c|c|c|c|c|c|c|c|c|}
\hline Authors, year & Country & $\begin{array}{l}\text { Location of } \\
\text { data } \\
\text { collection }\end{array}$ & $\begin{array}{c}\text { Total } \\
\text { number of } \\
\text { respondents } \\
\text { (Final } \\
\text { sample size) } \\
\end{array}$ & $\begin{array}{l}\text { Subjects' } \\
\text { age } \\
\text { (years) }\end{array}$ & Caries index & Socioeconomic indicator & Other measures & $\begin{array}{c}\text { Association between } \\
\text { socioeconomic } \\
\text { indicators and dental } \\
\text { caries }\end{array}$ & $\begin{array}{l}\text { Newcastle- } \\
\text { Ottawa scale }\end{array}$ \\
\hline $\begin{array}{c}\text { Holst \& } \\
\text { Schuller, } 2011 \\
{[63]}\end{array}$ & $\begin{array}{c}\text { Nord- } \\
\text { Trondelag }\end{array}$ & $\begin{array}{l}\text { Clinical } \\
\text { setting }\end{array}$ & $\begin{array}{c}\text { Two Birth- } \\
\text { cohorts in } \\
\text { age groups } \\
\text { between 35- } \\
44 \text { from } \\
1983 \text { to } \\
2006 \\
\text { Year } 1983= \\
500(300) \\
1994=350 \\
(135) \\
2006=250 \\
(158) \\
\text { (cross- } \\
\text { sectional } \\
\text { results over } \\
33 \text { years) }\end{array}$ & $\begin{array}{l}\text { Age- } \\
\text { group } \\
35-44 \\
\text { years }\end{array}$ & $\begin{array}{c}\text { DMFT } \\
\text { DMFS } \\
\text { Decayed surfaces- } \\
\text { DS } \\
\text { Decayed teeth-DT } \\
\text { Mean }\end{array}$ & $\begin{array}{l}\text { Education: quartile } \\
\text { (shortest education, } \\
\text { second shortest education, } \\
\text { second longest education, } \\
\text { longest education) }\end{array}$ & Age & $\begin{array}{l}1983 \text {-Less schooling } \\
\text { and more DS } \\
(p<0.05)^{*} \\
\text { 2006- education and } \\
\text { DMFS }(p>0.05) *\end{array}$ & $6(9)$ \\
\hline $\begin{array}{c}\text { Shearer et al., } \\
2012[61]\end{array}$ & $\begin{array}{c}\text { New } \\
\text { Zealand }\end{array}$ & Not reported & $\begin{array}{c}\text { Birth cohort } \\
\text { of } 1,037 \\
\text { children } \\
\text { born at the } \\
\text { queen Mary } \\
\text { Hospital } \\
932 \text { dentally } \\
\text { examined at } \\
\text { age } 32 \text { years } \\
\text { (626-had } \\
\text { both parents } \\
\text { interviewed } \\
\text { (complete } \\
\text { information) }\end{array}$ & 32 years & $\begin{array}{c}\text { DMFS } \\
\text { Decayed surface- } \\
\text { DS } \\
\text { Mean } \\
\text { DMFS }>20 \\
\text { DMFS }=12\end{array}$ & $\begin{array}{l}\text { Socioeconomic (SES): } \\
\text { low, medium, high }\end{array}$ & $\begin{array}{l}\text { Sex, use of dental } \\
\text { services, smoking } \\
\text { status, familial } \\
\text { risk, plaque } \\
\text { trajectory }\end{array}$ & $\begin{array}{c}\text { Less SES at age } 32 \\
\text { and more DMFS } \\
(p>0.05) * * \\
\text { Low SES-RR }=1.15 \\
(0.95-1.40) \\
\text { Medium SES } \\
\text { RR }=1.05 \\
(0.88-1.26)\end{array}$ & $8(9)$ \\
\hline
\end{tabular}

* Bivariate analysis; $* *$ multivariate analysis. 
Table 3. Study characteristics and results from ecological study.

\begin{tabular}{|c|c|c|c|c|c|c|c|c|c|}
\hline Authors, year & Country & $\begin{array}{l}\text { Location of } \\
\text { data } \\
\text { collection }\end{array}$ & $\begin{array}{c}\text { Total } \\
\text { number of } \\
\text { respondents } \\
\text { (Final } \\
\text { sample size) }\end{array}$ & $\begin{array}{c}\text { Subjects' } \\
\text { age } \\
\text { (years) }\end{array}$ & Caries index & Socioeconomic indicator & Other measures & $\begin{array}{l}\text { Association between } \\
\text { socioeconomic } \\
\text { indicators and dental } \\
\text { caries }\end{array}$ & $\begin{array}{l}\text { Newcastle- } \\
\text { Ottawa scale }\end{array}$ \\
\hline $\begin{array}{c}\text { Bernabe et al., } \\
2009 \text { [57] }\end{array}$ & 18 countries & $\begin{array}{c}\text { National } \\
\text { statistics on } \\
\text { dental caries } \\
\text { experience } \\
\text { obtained } \\
\text { from WHO } \\
\text { Oral Health } \\
\text { Country/Are } \\
\text { a Profile } \\
\text { Programme } \\
\text { (Surveys } \\
\text { conducted } \\
\text { between } \\
\text { 1995 and } \\
2005 \text { ) }\end{array}$ & $\begin{array}{c}\text { Ecological } \\
\text { data from } \\
\text { the } 50 \\
\text { richest } \\
\text { countries in } \\
\text { the world } \\
\text { (18 included } \\
\text { in the } \\
\text { analysis) }\end{array}$ & $\begin{array}{c}35 \text { to } \\
44 \text { years }\end{array}$ & $\begin{array}{c}\text { DMFT } \\
\text { Decayed teeth-DT }\end{array}$ & $\begin{array}{l}\text { Gross domestic product } \\
\text { per capita; } \\
\text { Gross national income per } \\
\text { capita in } 2000 \text { (in } \\
\text { dollars); } \\
\text { Gini coefficient }\end{array}$ & $\begin{array}{l}\text { Caries index, } \\
\text { restorative index, } \\
\text { treatment index }\end{array}$ & $\begin{array}{c}\text { Worse Gini } \\
\text { coefficient and more } \\
\text { decayed teeth } \\
(p>0.05)^{*}\end{array}$ & $5(9)$ \\
\hline
\end{tabular}

* Bivariate analysis. 


\subsection{Study Location and Language}

The language of the cross-sectional studies was predominantly English, although the studies were conducted in different countries, namely Germany, Finland, Italy, Burkina Faso (Africa), Norway, Brazil, the United States, Australia, Sweden, Israel, Lithuania, Portugal, Denmark, Iran, China, Lebanon and Canada. One cross-sectional study was published in Croatian and was translated prior to analysis [12] (Tables 1 and 2). The only ecological study included in the present study involved the analysis of health data from 18 different countries [57]. All studies were published between 1975 and 2012.

\subsection{Population Characteristics}

The studies involved populations in age groups between 19 and 60 years. In three studies, the participant ages were defined by a mean of 21 years [43], a minimum of 19 years [28], and a minimum of 50 years [38].

Twenty-five studies were population-based studies with randomization involving men and women, 17 of which defined the sample group prior to conducting the study [16-18,31,34-36,39-41,44,45,48, $49,51,53,55]$. Only nine studies reported that the sample group was representative of the population studied [16,34,39-41,48,51,53,55].

The studies by Brennan et al. [17,18] reported the analysis of data from a single epidemiological survey conducted in the city of Adelaide (southern Australia) in 2004-2005. However, the variables differed with regard to categorization. The studies by Celeste et al. $[53,55]$ also included the analysis of data from a single epidemiological survey conducted in 2002-2003, with the participation of 330 municipalities of Brazil.

Five studies evaluated samples of men recruited at a military base (convenience samples) $[12,37,43,46,47]$. The study by Sgan-Cohen et al. [43] included data from exams performed in consecutive years $(1994,1995,1996$ and 1997), with a total of 7,139 military participants.

The cross-sectional study by Schuller [36] analyzed data on the population of Oslo (Norway) from 1983 and 1994, describing statistically significant associations between the socioeconomic indicators and dental caries in both years. The ecological study addressed dental caries in 18 of the 50 richest countries in the world, namely Australia, Austria, Canada, Denmark, Finland, France, Germany, China, Ireland, Israel, Italy, Japan, Singapore, Slovenia, Spain, Sweden, the United Kingdom and the United States [57] (Table 3). The number of participants submitted to oral exams in the 38 studies ranged from 73 [30] to 20,695 [55].

\subsection{Measures of Dental Caries and Data Collection}

Different indices were identified, such as decayed, missing and filled teeth (DMFT); decayed, missing and filled surfaces (DMFS); decayed and filled teeth (DFT) and decayed and filled surfaces (DFS). Different diagnostic criteria for dental caries, such as criteria established by the World Health Organization (WHO) and criteria proposed by the US National Institute of Dental Research, were also identified. The following were the parameters used for the assessment of dental caries: 
$\checkmark$ Mean or median of the DMFT index and/or separate components [16-18,28-30,33,35,37, 39-44,46,47,49-51,53-57,60,63];

$\checkmark$ Mean or median of the DMFS index and/or separate components [30,32,34,36,39,40,45,52,54,61-63];

$\checkmark$ Mean number of surfaces with non-cavitated caries [52];

$\checkmark$ Mean number of teeth with root decay [64];

$\checkmark$ Mean number and percentage of functional teeth [28];

$\checkmark$ Quartiles of total number of decayed teeth and DMFT index (25, 50 and 75\%) [13];

$\checkmark$ Total number of decayed teeth and total number of decayed/filled teeth [38];

$\checkmark$ Number of decayed teeth, categorized as none, one to two decayed teeth and three or more decayed teeth [28];

$\checkmark$ DMFT, categorized as $\leq 21$ and $>21[16]$;

$\checkmark$ DMFS, categorized as $<10$ and $\geq 10$ [48];

$\checkmark$ DMFS, categorized as $>20$ [61];

$\checkmark$ DMFS, categorized as 12 [61];

$\checkmark$ Number of decayed surfaces, categorized as $<4$ and $\geq 4$ [40];

$\checkmark$ Number of decayed teeth, categorized as $\geq 7$ and $<7$ [31];

$\checkmark$ Number of decayed surfaces, categorized as $\geq 2$ and $<2$ [54];

$\checkmark$ Absence of new carious lesions or new restorations [59];

$\checkmark$ Number of decayed and filled root surfaces [40,59].

Nineteen studies reported using the WHO criteria for the oral exam [16,30,32,34,35,37, 39-44,46,47,49,51,53-55]. Regarding the exam location, the clinical setting was described in 18 studies $[12,17,18,28-30,33,37,39,42,45,47,48,50,52,54,60,63,64]$. In all studies, the exams for the diagnosis of dental caries were performed by dentists, with the exception of one study, in which the exams were performed by a dental hygienist [28].

In eight studies, X-ray exams were performed in tandem with clinical exams for the diagnosis of dental caries $[29,33,34,45,48,54,60,62]$. In the study by Bille [62], radiographs were taken at the homes of the subjects using a portable device. Only two studies reported using X-ray exams to calibrate the oral health professionals in their evaluation of bitewings [38,62]. However, it is unclear in the study by Unell et al. [38] whether the X-ray exam was used as a complementary exam for the diagnosis of carious lesions.

Regarding the calibration of the researchers for the clinical exam of the teeth, only 19 studies (46.34\%) described the Kappa index value or percentage of intra-examiner and/or inter-examiner agreement [32,34,35,39-44,47,48,51-55,60,62,63]. Kappa results ranged from 0.61 to 0.98 .

\subsection{Socioeconomic Indicators and Other Variables}

Different socioeconomic criteria were considered in the studies, demonstrating considerable diversity among the indices and criteria employed (Tables 1-3): schooling, literacy rate, school frequency, educational level (in years of study), socioeconomic status, social status, inequity regarding municipal revenue, social class, household income, income per capita, government benefits, satisfaction with income, occupation, employed population, unemployment and community indices, 
such as the Gini coefficient, which measures the degree of inequality in the distribution of individuals based on income per capita (ranging from 0 (absence of inequality) to 1 (maximal inequality)).

\subsection{Statistical Analysis of Associations between Dental Caries and Socioeconomic Indicators}

Twenty one out of the 41 studies employed multivariate statistical analyses, whereas 20 studies (48.78\%) only employed bivariate analyses with no adjustments for confounding variables. For the 21 studies that employed multivariate analyses, only 11 studies presented the results of both bivariate and multivariate analyses (Tables 1-3). Periodontal status, visits to the dentist, smoking habits, oral hygiene habits (brushing frequency, use of dental floss), gender, age and place of residence (urban or rural area) were used as confounding variables (Tables 1-3).

Fourteen studies used more than one socioeconomic variable, such as income and schooling, to assess associations with dental caries. Table 4 displays the quantitative distribution of the analyses performed on socioeconomic indicators by multivariate analysis and the associations: positive $(+)$ (worse socioeconomic indicator associated with a greater severity of dental caries; $95 \%$ CI does not include 1.0 or $p<0.05$ ); negative $(-)$ (worse socioeconomic indicator associated with a lesser severity of dental caries; 95\% CI does not include 1.0 or $p<0.05$ ); and null (socioeconomic indicator not associated with severity of dental caries; $95 \%$ CI including 1.0 or $p>0.05$ ). Besides $95 \%$ CI $p$-value was also considered because some studies reported results in $p$-value instead of $95 \% \mathrm{CI}$. The evaluation of the results of the analysis considered associations between socioeconomic determinants and the number of decayed or cavitated teeth. In the absence of the latter indicator, the evaluation considered the results of the DMFT, DMFS or other parameters used to determine the association with dental caries.

Table 4. Quantitative distribution of statistical analyses and type of association (positive $(+)$, negative (-) or null (\#)) according to socioeconomic indicators.

\begin{tabular}{|c|c|c|c|}
\hline Socioeconomic indicator & \multicolumn{3}{|c|}{$\begin{array}{c}\text { Multivariate analysis: Socioeconomic indicator and dental caries } \\
\text { OR }(95 \% \mathrm{CI}) \text { or Beta or } \mathbf{R}^{2} \text { or } R R\end{array}$} \\
\hline Type of association * & $\begin{array}{l}+(95 \% \text { CI does not } \\
\text { include } 1.0 \text { or } p<0.05)\end{array}$ & $\begin{array}{c}-(95 \% \text { CI does not } \\
\text { include } 1.0 \text { or } p<0.05)\end{array}$ & $\begin{array}{c}\#(95 \% \text { CI includes } \\
1.0 \text { or } p>0.05) \\
\end{array}$ \\
\hline $\begin{array}{c}\text { SCHOOLING } \\
\text { Schooling Subject's } \\
\text { Tervonen et al., } 1991 \text { [31] } \\
\text { Sgan-Cohen et al., } 1999 \text { [37] } \\
\text { Hahn et al., 1999 [64] } \\
\text { Unell et al., } 1999[38] \\
\text { Brodeur et al., } 2000[40] \\
\text { Sgan-Cohen et al., } 2000[43] \\
\text { Varenne et al., } 2006[49] \\
\text { Geyer et al., } 2010[16] \\
\text { Brennan et al., } 2011[18] \\
\text { Schooling Father's } \\
\text { Julihn et al., } 2006[48]\end{array}$ & $\begin{array}{l}\mathrm{OR}=1.12(1.03-1.20) \\
\mathrm{R}=-0.16(p<0.001) \\
(p<0.05) \\
(p<0.001) \\
\mathrm{OR}=2.95(1.52-5.74) \\
\mathrm{B}=-0.25(p<0.01)\end{array}$ & $\mathrm{OR}=2.99(p<0.05)$ & $\begin{array}{l}(p>0.05) \\
\mathrm{OR}=1.2(0.79-1.81)\end{array}$ \\
\hline
\end{tabular}


Table 4. Cont.

\begin{tabular}{|c|c|c|c|}
\hline Socioeconomic indicator & \multicolumn{3}{|c|}{$\begin{array}{c}\text { Multivariate analysis: Socioeconomic indicator and dental caries } \\
\text { OR }(95 \% \mathrm{CI}) \text { or Beta or } \mathrm{R}^{2} \text { or } \mathrm{RR}\end{array}$} \\
\hline Type of association * & $\begin{array}{l}+(95 \% \text { CI does not } \\
\text { include } 1.0 \text { or } p<0.05)\end{array}$ & $\begin{array}{l}-(95 \% \text { CI does not } \\
\text { include } 1.0 \text { or } p<0.05)\end{array}$ & $\begin{array}{l}\#(95 \% \text { CI includes } \\
1.0 \text { or } p>0.05)\end{array}$ \\
\hline $\begin{array}{c}\text { SUBJECT'S INCOME } \\
\text { Brodeur et al., } 2000 \text { [40] } \\
\text { Gilbert et al., } 2001 \text { [59] } \\
\text { Brennan et al., } 2007 \text { [50] } \\
\text { Skudutyte-Rysstad et al., } \\
2009 \text { [54] } \\
\text { Brennan et al., } 2010 \text { [17] } \\
\text { Geyer et al., } 2010[16] \\
\text { Brennan et al., } 2011 \text { [18] }\end{array}$ & $\begin{array}{l}\mathrm{OR}=3.8(2.19-6.48) \\
\mathrm{OR}=2.5(p<0.05) \\
(p<0.001) \\
\mathrm{OR}=4.5(1.9-10.2) \\
(p<0.01) \\
\mathrm{OR}=2.34(1.00-5.55) \\
\text { Beta }=-0.27(p<0.001)\end{array}$ & & \\
\hline $\begin{array}{c}\text { OCCUPATION/JOB } \\
\text { Occupation/Subject's job } \\
\text { Unell et al., } 1999 \text { [38] } \\
\text { Varenne et al., } 2006 \text { [49] } \\
\text { Roberts-Thomson et al., } \\
2008 \text { [52] } \\
\text { Occupation/Father's job } \\
\text { Julihn et al., } 2006 \text { [48] } \\
\text { Occupation/Mother's job } \\
\text { Julihn et al., } 2006 \text { [48] }\end{array}$ & $\begin{array}{l}(p<0.05) \\
(p<0.05)\end{array}$ & $\mathrm{OR}=5.26(p<0.05)$ & $(p>0.05)$ \\
\hline $\begin{array}{c}\text { SOCIOECONOMIC } \\
\text { STATUS-SES } \\
\text { SES Subject's } \\
\text { Marcenes \& Sheiham, } 1992 \\
\text { [32] } \\
\text { Berset et al., } 1996[34] \\
\text { Geyer et al., } 2010[16] \\
\text { Shearer et al., } 2012[61] \\
\text { Subject's SES trajectory } \\
\text { Thomson } \text { et al., } 2004[58]\end{array}$ & $\begin{array}{c}\mathrm{R}^{2}=0.35 \\
\mathrm{OR}=6.06(2.06-17.87) \\
(p<0.05)\end{array}$ & & $(p>0.05)$ \\
\hline $\begin{array}{l}\text { SOCIAL INDEX-Gini } \\
\text { Celeste et al., } 2009 \text { [53] } \\
\text { Celeste et al., } 2011 \text { [55] }\end{array}$ & $\begin{array}{l}\mathrm{OR}=2.49(2.30-2.68) \\
(p<0.05)\end{array}$ & & \\
\hline
\end{tabular}

$*(+)$ worse socioeconomic indicator significantly associated to higher severity of dental caries, $(-)$ worse socioeconomic indicator significantly associated to lower severity of dental caries negative association, (\#) no significantly association between socioeconomic indicator and dental caries.

Socioeconomic indicators were categorized as follows: schooling of the subject, income of the subject, occupation (of the subject or the subject's parents, recipient of government benefits), 
socioeconomic status (of the subject and socioeconomic trajectory) and social index (Gini coefficient) (Table 4).

In the analysis of the quantitative distribution of the statistical analyses of associations between socioeconomic indicators and dental caries, schooling of the subject was the most frequently used socioeconomic indicator. Lower schooling was statistically associated with greater severity of dental caries in six out of nine multivariate analyses. One study found that lower schooling was associated to lower severity of dental caries, two did not find significant association and one did not find association of schooling of the father and dental caries.

Seven studies analyzed income of the subject and all found that lower income of the subject was significantly associated to greater severity of dental caries.

Six studies addressed occupation. Two studies found significant association of better occupation of the subject and lower severity of caries; one found the contrary, the better occupation of the subject was significantly associated to higher severity of dental caries. Occupation of the father, mother and recipient of government benefits did not present association with dental caries.

Lower socioeconomic status of the subject was significantly associated to greater severity of dental caries in two out of two studies. Other two studies did not find significant association. Subject's socioeconomic trajectory, was significantly associated to dental caries in one study. That means that the low-low and high-low socioeconomic trajectory was significantly associated to more decayed surfaces.

The Gini coefficient was addressed in two studies and they presented that higher scores of Gini coefficient (more vulnerable) was significantly associated with higher severity of dental caries.

\section{Discussion and Conclusions}

\subsection{Strengths and Weaknesses of the Review}

This systematic review involved the search of multiple electronic databases, with no restrictions regarding language or year of publication. The reference lists of literature reviews were searched for other studies that could also be included. However, it was not possible to search technical reports, papers from research groups or committees and preprints. This could have accounted for some publication bias. One hundred and eighty nine studies were selected for full text analysis in which socioeconomic indicator could have hidden inside the paper as confounder but not as main subject. Efforts were made to try to find studies that reported socioeconomic indicators as risk factors of dental caries.

Due to the permanent nature of socioeconomic indicators, studies that evaluate such indicators tend to be observational rather than interventional. No clinical trials were found although the searches were conducted in three databases related to clinical trials (Controlled-Trial Database, Clinical Trials and the National Institute for Health and Clinical Excellence).

Most studies (65.85\%) were published after the year 2000. Cross-sectional studies were the most common (80.49\%). Seven investigations (17.07\%) were cohort studies, and one investigation (2.44\%) was an ecological study. Among the cohort studies (Table 2), three presented cross-sectional data [62-64], one presented data from the start of a prospective longitudinal study [64] and the other presented data on dental caries (DMFT index) at 13 and 20 years of age [62], with only the data at age 20 considered 
in the analysis. The study of Holst and Schuller [63] presented data from two birth-cohort (1959-1960 and 1929-1938), comprising the ages of 23-24, 45-54 and 35-44 years in 1983; 34-35, 55-64 and 35-44 years in 1994; and 46-47, 68-77 and 35-44 years in 2006. In this study only data from 35-44 years was analyzed. No case-control studies were found. These findings demonstrate a considerable tendency toward conducting and publishing cross-sectional studies and highlight the need for further case-control studies of incident cases, which offer greater scientific evidence through better control of possible methodological biases and data analysis.

Dental caries is still a health problem in most industrialized countries. In European countries during the XIXth century, rich individuals had more access to sugars and for that reason they had more dental caries. With industrialization there was an increasing provision and consumption of sugar for all populations, not only rich persons. In contrast, it has been observed a decline of dental caries in most industrialized countries over the past 20 years, as a result of a number of public health measures and use of fluorides [65]. However, these measures can be more accessible by individuals of higher socioeconomic status. This can be confirmed by the results, in which six studies observed that subjects with higher income had lower severity of dental caries. In fact, income can give more access to dental services, to fluoridated water, to oral products (toothbrushes and fluoridated toothpastes) and to information about oral health.

\subsection{Heterogeneity of Studies and Methodological Quality}

The diversity of socioeconomic indicators and the parameters used for the cutoff points for dental caries, years of schooling and income demonstrate the heterogeneity of the studies analyzed, thereby meta-analysis was not conducted in this review. The statistical method could render appropriate measure of the strength of the evidence and could assess bias what is a limitation of the review.

The most frequent socioeconomic indicators were schooling and income (household or per capita). There was several cut points used for schooling, which hinders comparisons between studies. Income was generally categorized as high/medium/low, which is somewhat subjective and depends on the definitions of upper, middle and lower class among different countries, as well as on differences in the income limits within each category and the exchange rate between the US dollar and the currency of the country of origin.

In addition to individual data, a small number of studies used collective indicators $[53,55,56]$, such as the Gini coefficient.

Socioeconomic status was the indicator with the greatest variation in its association with dental caries. The criteria used in each study to classify or group socioeconomic status were variable and subjective. For instance, the Brazilian ABA-Abipeme criteria [32] determine socioeconomic classification by attributing weights to items of domestic comfort and the level of schooling of the head of the family. The socioeconomic classification of the Brazilian population is divided into Classes A, B, C, D and E. One of the limitations of these criteria is the difficulty in comparing the results with findings from international studies because the indicator in question was designed for the Brazilian population. The ABA-Abipeme criteria consider the buying power of the population, which may not be relevant for countries in which access to consumer goods does not adequately portray socioeconomic status. 
There was marked heterogeneity in the criteria used for the evaluation of dental caries, although the majority of studies (68.29\%) employed either the mean or median DMFT index and/or its components. The mean or median DMFS was employed in $29.26 \%$ of the studies. Other indicators were also used, such as DMFT and DMFS severity.

The wide variety of population characteristics, age groups and criteria employed in clinical exams hinders the evaluation of the evidence. The large age range of the participants in the studies analyzed (19 to 60 years) may lead to varying results because dental caries are cumulative throughout life. Moreover, the most commonly used indices in the studies (DMFT and DMFS) consider both the past and present history of dental caries. The age group proposed by the WHO for studies on adults (35 to 44 years) was used in only 15 studies.

\subsection{Statement of Principal Findings}

The strength of the evidence included in the present review was affected by a number of methodological issues. Despite the heterogeneity of the socioeconomic indicators, there was scientific evidence of associations between dental caries and the subjects' schooling, income, and occupation, as well as the Gini coefficient. A lower level of schooling was associated with more dental caries in the statistical analyses that addressed the subjects' schooling. The subjects' occupation was associated with lower severity of dental caries, whereas the parents' occupation presented no association with dental caries. These findings were expected from the adult population because the influence of one's mother and father is more appropriately evaluated in studies involving children or adolescents. Subjects' income was also associated with dental caries, although the criteria for the assessment of income differed among the different studies (in terms of currency in each country and the exchange rate used for the conversion into dollars).

Socioeconomic status demonstrated considerable variability in the results of the association with dental caries. It is possible that the classifications used for this variable affected this finding. Although socioeconomic status is generally classified as high, medium, or low, this subjective classification depends on the researcher's assessment.

Among the studies that employed the Gini coefficient, two analyses were statistically associated to dental caries. The analysis of economic inequality between countries is mainly based on the interpretation of this coefficient, which is widely used in the literature because it reveals the degree of inequality in the distribution of income in a specific setting [66]. The Gini index considers information regarding the mean income of heads of families and compares the proportion of the total income of a portion of the population in relation to the weight of this subpopulation in the general population [67]. Therefore, the Gini coefficient evaluates the concentration of income without considering the social factor of schooling. In other words, it only evaluates the economic determinant. This index should therefore be used together with other indices to assess social determinants. For example, the Human Development Index is an international indicator that considers education, income and longevity. This index can be used as a complement to the Gini index because it also has limitations and has been criticized for its inadequate treatment of income, lack of comparability between survey years and different assessments of development between groups of countries [68]. 
Only 21 studies out of 41 studies employed multivariate analyses, and the remaining studies failed to adjust for confounding variables. The use of bivariate analysis alone can result in biased results regarding associations between socioeconomic indicators and dental caries. These studies are described in Tables 1-3 and highlighted the importance of the adjustment for confounding variables in studies. Multivariate analysis becomes important since the theoretical models of social determinants of health [69], and theoretical models of the determinants of dental caries [2,9] have measured social determinants of health at different levels of society [7].

The quality of the studies ranged between five and nine points, which demonstrates methodological variability. Most of the investigations were cross-sectional studies. This type of design offers a lower degree of scientific evidence compared with case-control and cohort studies. Regarding the NewcastleOttawa quality assessment scale, lower scores were mainly related to comparability (lack of a multivariate analysis), non-response rate and ascertainment of exposure (non-blinded interview). However, the use of scales for quality assessment has limitations that should be considered. The scales use a summary score that involves weights to different items and it is difficult to justify the weights assigned. Some authors have considered them of unreliable validity and less transparent to the users of the reviews [70]. Besides that, the use of the scale and weight criteria can be very subjective among reviewers. On the other hand, the Newcastle-Ottawa scale has been used to assess quality and it is able to standardize the scores among all studies. Newcastle-Ottawa scale was used for scoring studies, but methodological analysis of the studies was not based on it. Instead it was used narrative approach that could be fully reported by the present review.

The lack of participants in the oral exam (i.e., a lower number of individuals examined in relation to the total number of participants) was another negative aspect. Only nine studies $(21.95 \%)$ did not experience this loss. The non-participation of individuals in exams can result in data that do not adequately portray the population because there is no way of knowing whether those who refused to undergo the exam have a better or worse oral health status. The final important issue is the lack of external validity. Many of the studies offered no information regarding the sample size calculation and/or the analyzed population constituted a convenience sample.

\subsection{Suggestions for Further Research}

There is evidence of the association between socioeconomic indicators and dental caries in adults. However, the magnitude of evidence needs to be further evaluated. The strength of evidence could be hard to evaluate because it needed to know if a study adjusted the association for a true confounding factor or for mediators. There is a need for theoretical model to identify the role of each variable and it should be very clear throughout the study.

Studies that evaluate dental caries in subjects with wide range of age should perform adjusted analysis as odds ratios for controlling the age. The measurement of dental caries and socioeconomic indicators should be performed by different researchers to avoid or minimize the influence of these indicators on responses and on possibly biasing the results.

In conclusion, the findings of the present systematic review provide evidence that worse socioeconomic indicators, such as subject's schooling, income, occupation and the Gini coefficient, are associated with a greater severity of dental caries in adults. There was considerable degree of 
heterogeneity in the methodology, socioeconomic indicators and classification of dental caries was found in the studies analyzed. More cohort and case-control studies of incidence cases are needed to establish the magnitude of the scientific evidence regarding the association between socioeconomic indicators and dental caries.

\section{Acknowledgments}

This study was supported by the State of Minas Gerais Research Foundation (FAPEMIG), Pró-reitoria de Pesquisa da UFMG and the Brazilian Coordination of Higher Education (CAPES).

\section{Conflict of Interest}

The authors declare no conflict of interest.

\section{References}

1. Marthaler, T.M. Changes in dental caries 1953-2003. Caries Res. 2004, 38, 173-181.

2. Petersen, P.E. Sociobehavioural risk factors in dental caries-International perspectives. Community Dent. Oral Epidemiol. 2005, 33, 274-279.

3. van Nieuwenhuysen, J.P.; Carvalho, J.C.; D'Hoore, W. Status of dental caries in Belgium and neighboring countries. Rev. Belge Med. Dent. 2002, 57, 186-205.

4. Tickle, M. The 80:20 phenomenon: Help or hindrance to planning caries prevention programmes? Community Dent. Health 2002, 19, 39-42.

5. Narvai, P.C.; Frazão, P.; Roncalli, A.G.; Antunes, J.L.F. Cárie dentária no Brasil: Declínio, polarização, iniqüidade e exclusão social. Rev. Panam Salud Publica 2006, 19, 385-393.

6. Berkman, L.F. Social epidemiology: Social determinants of health in the United States: Are we losing ground? Annu Rev. Public Health 2009, 30, 27-41.

7. Krieger, N. Theories for social epidemiology in the 21 st century: An ecosocial perspective. Int J. Epidemiol. 2001, 30, 668-677.

8. Rothman, K.J.; Stein, Z.; Susser, M. Rebuilding bridges: What is the real role of social class in disease occurrence? Eur. J. Epidemiol. 2011, 26, 431-432.

9. Holst, D.; Schuller, A.A.; Aleksejuniené, J.; Eriksen, H.M. Caries in population-A theoretical, causal approach. Eur. J. Oral Sci. 2001, 109, 143-148.

10. Luglie, P.F.; Cellai, A.; Cossi, R.; Chessa, G. Dental caries and socioeconomic development of Sardinian population. Minerva Stomatol. 1989, 38, 673-678.

11. Ogunbodede, E.O.; Olusile, A.O.; Ogunniyi, S.O.; Faleyimu, B.L. Socio-economic factors and dental health in an obstetric population. West. Afr. J. Med. 1996, 15, 158-162.

12. Badel, T.; Restek-Despotusic, S.; Kern, J.; Keros, J.; Segovic, S. Caries in croatian army recruits in 2000. Acta Med. Croat. 2006, 60, 315-318.

13. Bastos, J.L.; Nomura, L.H.; Peres, M.A. Dental caries and associated factors among young male adults between 1999 and 2003 in Southern Brazil. Community Dent. Health 2007, 24, 122-127.

14. Crossner, C.G.; Unell, L. A longitudinal study of dental health from the age of 14 to 41. Swed. Dent. J. 2007, 31, 65-74. 
15. Hopcraft, M.S.; Morgan, M.V. Pattern of dental caries experience on tooth surfaces in an adult population. Community Dent. Oral Epidemiol. 2006, 34, 174-183.

16. Geyer, S.; Schneller, T.; Micheelis, W. Social gradients and cumulative effects of income and education on dental health in the fourth German oral health study. Community Dent. Oral Epidemiol. 2010, 38, 120-128.

17. Brennan, D.; Spencer, J.; Roberts-Thomson, K. Dental knowledge and oral health among middleaged adults. Aust. N. Z. J. Public Health 2010, 34, 472-475.

18. Brennan, D.S.; Spencer, A.J.; Roberts-Thomson, K.F. Dental self-care and visiting behaviour in relation to social inequality in caries experience. Community Dent. Health 2011, 28, 216-221.

19. Bandeca, M.C.; Nadalin, M.R.; Calixto, L.R.; Saad, J.R.; Silva, S.R. Correlation between oral health perception and clinical factors in a Brazilian community. Community Dent. Health 2011, 28, 64-68.

20. Nau, J.Y. The sad dental state of French peasants. Rev. Med. Suisse 2011, 7, 1834-1835.

21. Pierleoni, P.; Fazio, D. Epidemiologic study of dental caries in a group of soldiers from the Alpine Regiment of Trentino-Alto Adige. Arch.Stomatol. 1979, 20, 205-215.

22. Reddy, V.R. Dental caries in the permanent teeth of the people of Gulbarga, Karnataka. Acta Anthropogenet. 1980, 4, 89-127.

23. Treldal, K.; Oustad, B. Dental health habits of 40-year old patients in Aust-Agder county. Dental status of non-recall patients. Nor. Tannlaegeforen Tid. 1990, 100, 328-332.

24. Berge, K.A.; Fylkesnes, K. Dental health and dental attendance among 25- and 40-year olds. Nor. Tannlaegeforen Tid. 1991, 101, 408-412.

25. Rao, S.; Singh, M.A.; Singh, B.P.; Rao, M.S. Oral health assessment of an adult rural community. J. Pierre Fauchard Acad. 1994, 8, 15-19.

26. Murariu, A., Danila, I., Hanganu, C. Dental caries in adults, 35 to 44 years, Iasi county. Rev. Med. Chir. Soc. Med. Nat. Iasi. 2007, 111, 482-486.

27. Wells, G.A.; Shea, B.; O’Connell, D.; Peterson, J.; Welch, V.; Losos, M.; Tugwell, P. The Newcastle-Ottawa Scale (NOS) for Assessing the Quality of Nonrandomized Studies in MetaAnalyses. Available online: http://www.ohri.ca/programs/clinical_epidemiology/oxford.asp (accessed on 9 September 2012).

28. Nikias, M.K.; Fink, R.; Shapiro, S. Comparisons of poverty and nonpoverty groups on dental status, needs, and practices. J. Public Health Dent. 1975, 35, 237-259.

29. Hansen, B.F. Caries experience in a Norwegian urban population. Community Dent. Oral Epidemiol. 1977, 5, 132-135.

30. Meyer, K.; Freitas, E.; Davis, R.K.; Freitas, J.; Kristoffersen, T. Dental health among young adult Portuguese in relation to socio-economic differences. Rev. Port. Estomatol Cir. Maxilofac. 1983, 24, 461-478.

31. Tervonen, T.; Knuuttila, M.; Nieminen, P. Risk factors associated with abundant dental caries and periodontal pocketing. Community Dent. Oral Epidemiol. 1991, 19, 82-87.

32. Marcenes, W.S.; Sheiham, A. The relationship between work stress and oral health status. Soc. Sci Med. 1992, 35, 1511-1520. 
33. Eriksen, H.M.; Marques, M.D.; Bjertness, E.; Moe, B. Dental caries determinants in an adult Portuguese population and a comparison with Norwegian adults. Acta Odontol Scand. 1996, 54, 49-54.

34. Berset, G.P.; Eriksen, H.M.; Bjertness, E.; Hansen, B.F. Caries experience of 35-year-old Oslo residents and changes over a 20-year period. Community Dent. Health 1996, 13, 238-244.

35. Hescot, P.; Bourgeois, D.; Doury, J. Oral health in 35-44 year old adults in France. Int Dent. J. 1997, 47, 94-99.

36. Schuller, A.A. Better oral health, more inequality-Empirical analysis among young adults. Community Dent. Health 1999, 16, 154-159.

37. Sgan-Cohen, H.D.; Horev, T.; Zusman, S.P.; Katz, J.; Eldad, A. The prevalence and treatment of dental caries among Israeli permanent force military personnel. Mil. Med. 1999, 164, 562-565.

38. Unell, L.; Soderfeldt, B.; Halling, A.; Birkhed, D. Explanatory models for clinically determined and symptom-reported caries indicators in an adult population. Acta Odontol Scand. 1999, 57, $132-138$.

39. Aleksejuniene, J.; Eriksen, H.M.; Holst, D. Variation in caries and treatment experience in 35-44year-old Lithuanians. Community Dent. Oral Epidemiol. 2000, 28, 356-364.

40. Brodeur, J.M.; Payette, M.; Benigeri, M.; Gagnon, P.F.; Olivier, M.; Chabot, D. Dental caries in Quebec adults aged 35 to 44 years. J. Can. Dent. Assoc. 2000, 66, 374-379.

41. Doughan, B.; Kassak, K.; Bourgeois, D.M. Oral health status and treatment needs of 35-44-year old adults in Lebanon. Int Dent. J. 2000, 50, 395-399.

42. Skudutyte, R.; Aleksejuniene, J.; Eriksen, H.M. Dental caries in adult Lithuanians. Acta Odontol. Scand. 2000, 58, 143-147.

43. Sgan-Cohen, H.D.; Katz, J.; Horev, T.; Dinte, A.; Eldad, A. Trends in caries and associated variables among young Israeli adults over 5 decades. Community Dent. Oral Epidemiol. 2000, 28, 234-240.

44. Lin, H.C.; Wong, M.C.; Zhang, H.G.; Lo, E.C.; Schwarz, E. Coronal and root caries in Southern Chinese adults. J. Dent. Res. 2001, 80, 1475-1479.

45. Paulander, J.; Axelsson, P.; Lindhe, J. Association between level of education and oral health status in 35-, 50-, 65- and 75-year-olds. J. Clin. Periodontol. 2003, 30, 697-704.

46. Ceylan, S.; Acikel, C.H.; Okcu, K.M.; Kilic, S.; Tekbas, O.F.; Ortakoglu, K. Evaluation of the dental health of the young adult male population in Turkey. Mil. Med. 2004, 169, 885-889.

47. Senna, A.; Campus, G.; Gagliani, M.; Strohmenger, L. Socio-economic influence on caries experience and CPITN values among a group of Italian call-up soldiers and cadets. Oral Health Prev. Dent. 2005, 3, 39-46.

48. Julihn, A.; Barr, A.M.; Grindefjord, M.; Modeer, T. Risk factors and risk indicators associated with high caries experience in Swedish 19-year-olds. Acta Odontol. Scand. 2006, 64, 267-273.

49. Varenne, B.; Petersen, P.E.; Ouattara, S. Oral health behaviour of children and adults in urban and rural areas of Burkina Faso, Africa. Int. Dent. J. 2006, 56, 61-70.

50. Brennan, D.S.; Spencer, A.J.; Roberts-Thomson, K.F. Caries experience among 45-54 year olds in Adelaide, South Australia. Aust. Dent. J. 2007, 52, 122-127.

51. Hessari, H.; Vehkalahti, M.M.; Eghbal, M.J.; Murtomaa, H.T. Oral health among 35- to 44-yearold Iranians. Med. Princ. Prac.t 2007, 16, 280-285. 
52. Roberts-Thomson, K.; Stewart, J.F. Risk indicators of caries experience among young adults. Aust. Dent. J. 2008, 53, 122-127.

53. Celeste, R.K.; Nadanovsky, P.; Leon, P.L.; Fritzell, J. The individual and contextual pathways between oral health and income inequality in Brazilian adolescents and adults. Soc. Sci. Med. 2009, 69, 1468-1475.

54. Skudutyte-Rysstad, R.; Sandvik, L.; Aleksejuniene, J.; Eriksen, H.M. Dental health and disease determinants among 35-year-olds in Oslo, Norway. Acta Odontol. Scand. 2009, 67, 50-56.

55. Celeste, R.K.; Fritzell, J.; Nadanovsky, P. The relationship between levels of income inequality and dental caries and periodontal diseases. Cad. Saude Publica 2011, 27, 1111-1120.

56. Chandra Shekar, B.R.; Reddy, C. Oral health status in relation to socioeconomic factors among the municipal employees of Mysore city. Indian J. Dent. Res. 2011, 22, 410-418.

57. Bernabe, E.; Sheiham, A.; Sabbah, W. Income, income inequality, dental caries and dental care levels: An ecological study in rich countries. Caries Res. 2009, 43, 294-301.

58. Thomson, W.M.; Poulton, R.; Milne, B.J.; Caspi, A.; Broughton, J.R.; Ayers, K.M. Socioeconomic inequalities in oral health in childhood and adulthood in a birth cohort. Community Dent. Oral Epidemiol. 2004, 32, 345-353.

59. Gilbert, G.H.; Duncan, R.P.; Dolan, T.A.; Foerster, U. Twenty-four month incidence of root caries among a diverse group of adults. Caries Res. 2001, 35, 366-375.

60. Bjertness, E.; Eriksen, H.M.; Hansen, B.F. Factors of importance for changes in dental caries among adults. A follow-up study of Oslo citizens from the age of 35 to 50 years. Acta Odontol. Scand. 1992, 50, 193-200.

61. Shearer, D.M.; Thomson, W.M.; Caspi, A.; Moffitt, T.E.; Broadbent, J.M.; Poulton, R. Family history and oral health: Findings from the Dunedin Study. Community Dent.Oral Epidemiol. 2012, 40, 105-115.

62. Bille, J. Dental caries in a group of 20 -year-olds after previous participation in public child dental health services in Copenhagen, Denmark. Community Dent. Oral Epidemiol. 1980, 8, 27-32.

63. Holst, D.; Schuller, A.A. Equality in adults' oral health in Norway. Cohort and cross-sectional results over 33 years. Community Dent. Oral. Epidemiol. 2011, 39, 488-497.

64. Hahn, P.; Reinhardt, D.; Schaller, H.G.; Hellwig, E. Root lesions in a group of 50-60 year-old Germans related to clinical and social factors. Clin Oral Investig. 1999, 3, 168-174.

65. Petersen, P.E.; Bourgeois, D.; Ogawa, H.; Day-Estupinan, S.; Ndiaye, C. The global burden of diseases and risk to oral health. Bull. Word Health Organ. 2005, 83, 66-69.

66. Barros, R.P.; Henriques, R.; Mendonca, R. Desigualdade e pobreza no Brasil: Retrato de uma estabilidade inaceitável. Rev. Bras. Ci Soc. 2000, 15, 123-142.

67. Junqueira, S.R.; Araújo, M.E.; Antunes, J.L.F.; Narvai, P.C. Indicadores socioeconômicos e recursos odontológicos em Municípios do Estado de São Paulo, Brasil, no final do século XX. Epidemiol Serv Saude 2006, 15, 41-53 (in Portuguese).

68. McGillivray, M.; Howard, W. Measuring development? The UNDP's human development index. J. Int. Dev. 2006, 5, 183-192.

69. Dahlgren, G.; Whitehead, M. Policies and Strategies to Promote Social Equity in Health. Available online: http://whaqlibdoc.who.int/euro/-1993/EUR_ICP_RPD414(2) (accessed on 15 October 2011). 
70. Higgins, J.P.T.; Altman, D.G. Chapter 8: Assessing Risk of Bias in Included Studies.In Cochrane Handbook for Systematic Reviews of Interventions; John Wiley \& Sons Ltd.: London, UK, 2008.

(C) 2012 by the authors; licensee MDPI, Basel, Switzerland. This article is an open access article distributed under the terms and conditions of the Creative Commons Attribution license (http://creativecommons.org/licenses/by/3.0/). 